NOTAS 



\title{
PUBLICACIONES SOBRE FILOLOGÍA INGLESA EN ESPAÑA (2015)
}

\author{
RESUMEN \\ Bibliografía relativa a Filología Inglesa publicada en España en 2015. \\ PALABRAS CLAVE: Filología Inglesa; bibliografía; España. \\ Abstract \\ Bibliography on English Studies published in Spanish, 2015. \\ KEY WORDS: English Studies; bibliography; Spain.
}

\section{Índice general}
A. LITERATURA INGLESA E IRLANDESA
B. LITERATURA NORTEAMERICANA
C. OTRAS LITERATURAS EN LENGUA INGLESA
D. ENFOQUES TEÓRICOS O GENERALISTAS
E. ESTUDIOS DE TRADUCCIÓN
F. CULTURA, CINE Y TELEVISIÓN 



\title{
A. LITERATURA INGLESA E IRLANDESA
}

\author{
Dídac Llorens Cubedo \\ UNED \\ dllorens@ flog.uned.es \\ MARIÁNGEl Soláns GARCía \\ UNED \\ masolans@madrid.uned.es
}

\section{Índice}

1. Literatura anglosajona, medieval y renacentista

2. Restauración y siglo XVIII

3. Siglo XIX

4. Siglo XX y época contemporánea

\section{LITERATURA ANGLOSAJONA, MEDIEVAL Y RENACENTISTA}

Bueno Alonso, Jorge Luis. "That's what I like about Shakespeare; it's the Pictures': A TeachingOriented Approach to Shakespearean Textual Analysis through Film (with Special Reference to Macbeth V.5.15-28)». Babel afial: Aspectos de filología inglesa y alemana 24 (2015): 131-144.

Calbi, Maurizio. «Exilic/Idyllic Shakespeare: Reiterating Pericles in Jacques Rivette's Paris nous appartient». The SEDERI Yearbook 25 (2015): 11-30.

Calvo, Clara. «Shakespeare in Khaki». English and American Studies in Spain: New Developments and Trends. Eds. Alberto Lázaro Lafuente y María Dolores Porto Requejo. Alcalá de Henares: Servicio de Publicaciones de la Universidad de Alcalá, 2015 (libro electrónico). 12-30.

CAporicci, Camilla. «The Tyranny of Immaterialism: Refusing the Body in The Winter's Tale». The SEDERI Yearbook 25 (2015): 31-54.

De Lorenzo, Eusebio. «Fantasmal Shkspr». Fantasmas, aparecidos y muertos sin descanso. Eds. Mercedes Aguirre, Cristina Delgado y Ana González-Rivas. Madrid: Abada, 2015. 201-216.

Dumas, María. «Imágenes del autor en la literatura vernácula medieval: el caso de la narrativa anglonormanda». Epos. Revista de filología 31 (2015): 405-420.

HAdFIEld, ANDREw. «Grimalkin and Other Shakespearean Celts». The SEDERI Yearbook 25 (2015): $55-76$.

Houliston, Victor. «Filling in the Blanks: Catholic Hopes for the English Succession». The SEDERI Yearbook 25 (2015): 77-104.

McKenzie, Clayton. «Of Death and Dukes: King Henry VI Part 2 and the "Danse Macabre"». Journal of English Studies 13 (2015): 71-82. 
Pedrosa Bartolomé, José Manuel. «El exemplo 51 de El Conde Lucanor (ATU 757), Mio Cid y King Lear: soberbia, ira, y el noble al que cierran las puertas de su casa». Revista de poética medieval 29 (2015): 263-290.

Perni Llorente, Remedios. «El año de Ricardo and the Degeneration of Europe». The Grove: Working Papers on English Studies 22 (2015): 135-147.

— «Reviviendo a Shakespeare: restos y montajes». Odisea 16 (2015): 143-158.

Pujante, Ángel-Luis, y Cerdà, JuAn F., ed. Shakespeare en España. Bibliografía anotada bilingüe. Murcia: Editum, 2015.

Rayner, Francesca. «Adapting Macbeth in a Lusophone Context: The Challenges of Intercultural Performance». The SEDERI Yearbook 25 (2015): 129-151.

Rodríguez Herrera, José Manuel «Shakespeare's Legal Wit: Evolution of the Translation of Shakespeare's Legal Puns into Spanish from the 20th to the 21st Century». Revista alicantina de Estudios Ingleses 28 (2015): 165-181.

Sell, Roger D. «Pinter, Herbert, Dickens: Post-postmodern Communicational Studies and the Humanities». English and American Studies in Spain: New Developments and Trends. Eds. Alberto Lázaro Lafuente y María Dolores Porto Requejo. Alcalá de Henares: Servicio de Publicaciones de la Universidad de Alcalá, 2015 (libro electrónico). 31-43.

ShaKespeare, William. Dramas históricos. Ed. Ángel-Luis Pujante. Madrid: Espasa, 2015.

\section{RESTAURACIÓN Y SIGLO XVIII}

Figueroa Dorrego, Jorge. «'Zounds, what Stuff's here?': The 'slight Farce' of Aphra Behn's The False Count». English and American Studies in Spain: New Developments and Trends. Eds. Alberto Lázaro Lafuente y María Dolores Porto Requejo. Alcalá de Henares: Servicio de Publicaciones de la Universidad de Alcalá, 2015 (libro electrónico). 84-90.

Lasa Álvarez, Begoña. «Constructing a Portrait of the Early-modern Woman Writer for Eighteenthcentury Female Readers: George Ballard's Memoirs of Several Ladies of Great Britain (1752)». The SEDERI Yearbook 25 (2015): 105-127.

Manuel, CARme, ed. The Enlightened Child: Eighteenth-Century Literature for Children. Valencia: JPM Ediciones, 2015.

Mora, María José. «The Casting of Sancho in Durfey's The Comical History of Don Quixote, Parts I-II (1694)». The SEDERI Yearbook 25 (2015): 155-167.

Rodríguez Loro, Nora. «Calderon's and Wycherley's Dancing-masters». English and American Studies in Spain: New Developments and Trends. Eds. Alberto Lázaro Lafuente y María Dolores Porto Requejo. Alcalá de Henares: Servicio de Publicaciones de la Universidad de Alcalá, 2015 (libro electrónico). 136-143.

SÁnchez Ruiz, RAQuel. «George Ridpath's Use of Evaluative Adjectives as Manipulative and Persuasive Strategies During the War of the Spanish Succession (1710-1713)». Journal of English Studies 13 (2015): 109-134.

Torralbo Caballero, Juan De Dios. «'The greatest extasy or bliss': Realism, Subversion and Eroticism in The Dumb Virgin; or The Force of Imagination». Revista canaria de Estudios Ingleses 71 (2015): 143-162.

\section{SIGLO XIX}

Fernández Rodríguez, Carmen María. «Le Nègre Reconnaissant (1814): A Translemic Analysis of Maria Edgeworth's The Grateful Negro into French». Babel afial: Aspectos de filología inglesa y alemana 24 (2015): 91-108. 
Fernández Rodríguez, Carmen María. «'To Be Chosen, Not Offered': An Introduction to Sarah Harriet Burney's Traits of Nature (1812)». ES: Revista de filología inglesa 36 (2015): 129-144.

Gaskell, Elizabeth. Norte y sur (1855). Trad. Elizabeth Power, ed. María José Coperías. Madrid: Cátedra, 2015.

González García, Jonatan. «Un estudio sobre la recepción de la poesía traducida de William Wordsworth en la España de los años 20: 'Tintern Abbey' y 'Personal Talk'». Odisea 16 (2015): 59-82.

Guerrero García, María Cristina. «Los cuentos de Oscar Wilde desde una perspectiva folclórica: Búsqueda de la esencia irlandesa». Babel afial: Aspectos de filología inglesa y alemana 24 (2015): 19-36.

Lasa Álvarez, Begoña. «Novela inglesa y censura inquisitorial durante el reinado de Fernando VII: un expediente de 1815-1816». Traducción y censura: nuevas perspectivas. Eds. Gora Zaragoza Ninet, Juan José Martínez Sierra y José Javier Ávila-Cabrera. Quaderns de Filologia. Estudis Literaris 20 (2015): 145-161.

López-Varela Azcárate, Asunción. «El futuro de las narratologías híbridas en Alicia en el país de las maravillas». 1616. Anuario de literatura comparada 5 (2015): 137-162.

Martínez Navajas, Jesús. «La influencia de la narrativa de Joseph Conrad en el cine y el rock'n'roll: análisis de tres canciones inspiradas en Heart of Darkness». Epos: Revista de Filología 31 (2015): 453-74.

Miralles Martorell, Carmen. «Of Snake Ladies and Other Reptiles. The Subversive Transition from the Femme Fatale to the Godmother in Vernon Lee's 'Pince Alberic and The Snake Lady'». Babel afial: Aspectos de filología inglesa y alemana 24 (2015): 37-54.

Puchal Terol, Victoria. «El héroe Crusoe: modernización del mito de Ulises en el siglo XIX». Tycho. Revista de iniciación en la investigación del teatro clásico grecolatino y su tradición 3 (2015): 65-80.

Sell, Roger D. «Pinter, Herbert, Dickens: Post-postmodern Communicational Studies and the Humanities». English and American Studies in Spain: New Developments and Trends. Eds. Alberto Lázaro Lafuente y María Dolores Porto Requejo. Alcalá de Henares: Servicio de Publicaciones de la Universidad de Alcalá, 2015 (libro electrónico). 31-43.

Valero Redondo, María. «Giving Voice to the Subaltern: Ideology and Socio-Historical Factors in Andrea Arnold's Adaptation of Wuthering Heights». English and American Studies in Spain: New Developments and Trends. Eds. Alberto Lázaro Lafuente y María Dolores Porto Requejo. Alcalá de Henares: Servicio de Publicaciones de la Universidad de Alcalá, 2015 (libro electrónico). 144-150.

VILlar, José María M. «De musa ideal a presencia opresiva: sobre la dualidad fantasmal de Elizabeth Siddal en la vida y obra de Dante Gabriel Rossetti». Fantasmas, aparecidos y muertos sin descanso. Eds. Mercedes Aguirre, Cristina Delgado y Ana González-Rivas. Madrid: Abada, 2015. 249-266.

\section{SIGLO XX Y CONTEMPORÁNEA}

All-Garralah, Aiman Sanad. «Encounter of the Twain: Rudyard Kipling's 'The City of Brass'». Odisea 16 (2015): 159-178.

Alonso Jerez, Marta. «Innocence and Experience: From Mina Murray to Mina Harker in The League of Extraordinary Gentlemen». English and American Studies in Spain: New Developments and Trends. Eds. Alberto Lázaro Lafuente y María Dolores Porto Requejo. Alcalá de Henares: Servicio de Publicaciones de la Universidad de Alcalá, 2015 (libro electrónico). 52-59.

Asensio Peral, Germán. «Flann O’Brien's Creative Loophole». Estudios irlandeses. Journal of Irish Studies 10 (2015): 1-13. 
Asensio Peral, Germán. «The Origins of Flann O'Brien's At Swim-Two-Birds». ES: Revista de filología inglesa 36 (2015): 47-62.

Athanasiades, ANDREAS. «Reposessing Islam: Affective Identity and Islamic Fundamentalism in Hanif Kureishi». Indialogs. Spanish Journal of India Studies 2 (2015): 55-71.

Beckett, Samuel. Relatos y textos para nada. Ed. y trad. José Francisco Fernández. Valencia: JPM Ediciones, 2015.

Bermejo Moreno, Mario. «The Heroine's Sexual Maturity in Angela Carter's The Bloody Chamber». Babel afial: Aspectos de filología inglesa y alemana 24 (2015): 5-18.

Brina, Maximiliano. «La voz inconformista de Patrick Kavanagh». Estudios irlandeses. Journal of Irish Studies 10 (2015): 35-43.

Cantillo Lucuara, Mayron Estefan. «On Death, Loss, Mystery, and Other Existential Concerns in Colm Tóibín's The Blackwater Lightship (1999)». Epos. Revista de filología 31 (2015): 375-388.

Carregal Romero, José. «Sexuality and the Culture of Silence in Colm Tóibín's 'The Pearl Fishers'». Atlantis. Journal of the Spanish Association of Anglo-American Studies 37.1 (2015): 69-83.

Cerezo Moreno, Marta. «'Returning Home versus Movement without Return: A Levinasian Reading of John Banville's The Sea». Atlantis. Journal of the Spanish Association of Anglo-American Studies 37.1 (2015): 51-68.

Chi Chang, Tsu. «I Am Nobody: Fantasy and Identity in Neil Gaiman's The Graveyard Book». Journal of English Studies 13 (2015): 7-18.

Coperías Aguilar, María José. «El periodismo de trinchera como testigo de la Primera Guerra Mundial. El ejemplo de The Wipers Times». Letras desde la trinchera: testimonios literarios de la Primera Guerra Mundial. Eds. Carme Manuel e Ignacio Ramos. Valencia: Publicacions de la Universitat de València, 2015. 215-231.

Delattre, Elisabeth. «The Great War and All That in A Hundred Doors by Michael Longley». Estudios irlandeses. Journal of Irish Studies 10 (2015): 77-84.

De Mingo Izquierdo, Nieves. «The Discursive Use of Humour in the Description of British Premiership Perception: The Contribution of Sue Townsend». Revista canaria de Estudios Ingleses 70 (2015): 107-121.

Domínguez Ruiz, Beatriz. «J. R. R. Tolkien's Construction of Multiple Masculinities in The Lord of the Rings». Odisea 16 (2015): 23-38.

García Hernández, Silvia. «Hilary Mantel's Eight Months on Ghazzah Street: The Displacement of British Expatriates in Saudi Arabia». Atlantis. Journal of the Spanish Association of AngloAmerican Studies 37.1 (2015): 85-100.

- «Water in the Construction of Michèle Roberts's In-Between Characters». Revista canaria de Estudios Ingleses 71 (2015): 163-173.

Gómez Reus, TeresA. «El segundo campo de batalla. Testimonios de enfermeras angloamericanas en el frente». Letras desde la trinchera: testimonios literarios de la Primera Guerra Mundial. Eds. Carme Manuel e Ignacio Ramos. Valencia: Publicacions de la Universitat de València, 2015. 249-268.

González Chacón, María Del Mar. «Myths in Crisis? Marina Carr's Revision of Feminine Myths in Contemporary Irish Theatre». The Grove: Working Papers on English Studies 22 (2015): 59-68.

GonzÁlez PÉrez, Leticia. «Nature and Culture in Tony Harrison's The Trackers of Oxyrhynchus». Tycho. Revista de iniciación en la investigación del teatro clásico grecolatino y su tradición 3 (2015): 5-24.

Goonetileke, D.C.R.A. «Salman Rushdie's Midnight's Children, The Play as Text and Performance: An Introductory Note». Indialogs. Spanish Journal of India Studies 2 (2015): 119-123.

Gutiérrez Carreras, Pablo, Abradelo De Usera, María Isabel e Armada Manrique, Ignacio, (coords.) J. R. R. Tolkien. El árbol de las historias. Madrid: CEU Editores, 2015.

JERSONSKY, EVA. «El universo femenino en la prosa temprana de Samuel Beckett: hacia la autonomía y la mundialización de la literatura». Estudios irlandeses. Journal of Irish Studies 10 (2015): 85-94. 
Koziol, Slawomir. «Between a Butterfly and a Cathedral: The Question of Art in Brideshead Revisited by Evelyn Waugh». Miscelánea 52 (2015): 69-87.

Llorens Cubedo, DídAc. «La tierra baldía de T. S. Eliot como poema de posguerra». Letras desde la trinchera: testimonios literarios de la Primera Guerra Mundial. Eds. Carme Manuel e Ignacio Ramos. Valencia: Publicacions de la Universitat de València, 2015. 297-310.

Marín Ruiz, Ricardo. «Revisiting Rosinante: Reinterpretations of the Cervantine Character in Rosinante to the Road Again, Monsignor Quixote, and Travels with Charley». Epos: Revista de Filología 31 (2015): 437-52.

Martín González, Juan José. «Dark (Neo)Victorians: Race and the Empire in Belinda Starling's The Journal of Dora Damage and Barbara Chase-Riboud's Hottentot Venus». English and American Studies in Spain: New Developments and Trends. Eds. Alberto Lázaro Lafuente y María Dolores Porto Requejo. Alcalá de Henares: Servicio de Publicaciones de la Universidad de Alcalá, 2015 (libro electrónico). 113-120.

Martínez López, Miguel. «Las sombras de la Primera Guerra Mundial en la tempestad de Brave New World». Letras desde la trinchera: testimonios literarios de la Primera Guerra Mundial. Eds. Carme Manuel e Ignacio Ramos. Valencia: Publicacions de la Universitat de València, 2015. 405-416.

Martínez Luciano, Juan Vicente. «La poesía inglesa de la Primera Guerra Mundial». Letras desde la trinchera: testimonios literarios de la Primera Guerra Mundial. Eds. Carme Manuel e Ignacio Ramos. Valencia: Publicacions de la Universitat de València, 2015. 207-214.

Martínez Navajas, Jesús. «La influencia de la narrativa de Joseph Conrad en el cine y el rock'n'roll: análisis de tres canciones inspiradas en Heart of Darkness». Epos: Revista de Filología 31 (2015): 453-74.

Melia, Paul. «Imperial Orwell». Atlantis. Journal of the Spanish Association of Anglo-American Studies 37.2 (2015): 11-25.

Menéndez Cuesta, Vanesa. «'The mouths of corpses': Death, Femininity and the Grotesque in Sylvia Plath's Poetry». Odisea 16 (2015): 97-110.

Meseguer Cutillas, Purificación. «La traducción como arma propagandística: censura de Orwell, Abellio y Koestler en la España franquista». Traducción y censura: nuevas perspectivas. Eds. Gora Zaragoza Ninet, Juan José Martínez Sierra y José Javier Ávila-Cabrera. Quaderns de Filologia. Estudis Literaris 20 (2015): 107-122.

Morató, Yolanda. «Wyndham Lewis y su recepción en España: etapas editoriales y traducciones». Traducción y censura: nuevas perspectivas. Eds. Gora Zaragoza Ninet, Juan José Martínez Sierra y José Javier Ávila-Cabrera. Quaderns de Filologia. Estudis Literaris 20 (2015): 183-197.

Pellicer-Ortín, Silvia. «Linda Grant: An Interview». Atlantis. Journal of the Spanish Association of Anglo-American Studies 37.2 (2015): 203-217.

Peñalba García, Mercedes. «'My world is sight': H. G. Wells's Anti-utopian Imagination in 'The Country of the blind'». Epos. Revista de filología 31 (2015): 475-484.

PietrzaK, Wit. «Decalibrating the Language. J. H. Prynne's Biting the Air». Miscelánea 52 (2015): 103-118.

Reyes Torres, Agustín. «Juegos de soldados. La Gran Guerra en la literatura juvenil inglesa». Letras desde la trinchera: testimonios literarios de la Primera Guerra Mundial. Eds. Carme Manuel e Ignacio Ramos. Valencia: Publicacions de la Universitat de València, 2015. 269-277.

Rodríguez Álvarez, Belén. «The Girl on the Stairs de Louise Welsh: Conformando una nueva detective, deconstruyendo discursos normativos de género». Babel afial: Aspectos de filología inglesa y alemana 24 (2015): 73-90

Ryan, John Charles. «Tolkien's Sonic Trees and Perfumed Herbs: Plant Intelligence in Middleearth».Ecozon@. European Journal of Literature, Culture and Environment 6.2 (2015): 125-141.

SÁNCHEz, MÉLODY. «Understanding Scarlett Thomas' Fiction: The Anti-Heroic Heroine». The Grove: Working Papers on English Studies 22 (2015): 149-162. 
Sell, Roger D. «Pinter, Herbert, Dickens: Post-postmodern Communicational Studies and the Humanities». English and American Studies in Spain: New Developments and Trends. Eds. Alberto Lázaro Lafuente y María Dolores Porto Requejo. Alcalá de Henares: Servicio de Publicaciones de la Universidad de Alcalá, 2015 (libro electrónico). 31-43.

Solá Buil, Ricardo J. «Landscape in Detective Fiction. A View of Julian Symmons's Thirty First of February and The Players and the Game, P.D. James's Shroud for a Nitghingale and Sarah Dunant's Under my Skin». Verbeia. Revista de estudios filológicos 0 (2015): 319-332.

Sumillera, Rocío G. «Aflicciones mentales y sueños de guerra en Rose Macaulay, Rebecca West, Virginia Woolf and Robert Graves». Letras desde la trinchera: testimonios literarios de la Primera Guerra Mundial. Eds. Carme Manuel e Ignacio Ramos. Valencia: Publicacions de la Universitat de València, 2015. 233-247.

Valero Redondo, María. «Giving Voice to the Subaltern: Ideology and Socio-Historical Factors in Andrea Arnold's Adaptation of Wuthering Heights». English and American Studies in Spain: New Developments and Trends. Eds. Alberto Lázaro Lafuente y María Dolores Porto Requejo. Alcalá de Henares: Servicio de Publicaciones de la Universidad de Alcalá, 2015 (libro electrónico). 144-150.

VILlar Flor, Carlos. «Graham Greene and Leopoldo Durán: Quixotic Companions across Spain and Portugal». The Grove: Working Papers on English Studies 22 (2015): 213-228.

Villegas-López, Sonia. "'Memory lives inside us': Writing as Memory Traces in The Secret Staircase». Atlantis. Journal of the Spanish Association of Anglo-American Studies 37.2 (2015): 45-62.

Wallhead Salway, Celia Margaret. «Meditations on Genre in Salman Rushdie's Joseph Anton». Revista canaria de Estudios Ingleses 70 (2015): 89-104.

Wan Lih, AnN. «A Woman Alone: The Depiction of Spinsters in Irish Women's Short Stories». Estudios irlandeses. Journal of Irish Studies 10 (2015): 44-57.

Yebra Pertusa, José María. «Traumatic Identity and Aura in David Lodge's Author, Author». ES: Revista de filología inglesa 36 (2015): 167-184. 


\title{
B. LITERATURA NORTEAMERICANA
}

\author{
Mariángel Soláns García \\ UNED \\ masolans@madrid.uned.es \\ DídAC LloRens Cubedo \\ UNED \\ dllorens@ flog.uned.es
}

Albert-Llácer, Mercedes. «The Tomboy Goes West: An Exploration of Gender and Race in Barbara Kingsolver's The Bean Trees». English and American Studies in Spain: New Developments and Trends. Eds. Alberto Lázaro Lafuente y María Dolores Porto Requejo. Alcalá de Henares: Servicio de Publicaciones de la Universidad de Alcalá, 2015 (libro electrónico). 45-51.

Alsina Rísquez, Cristina. «Un mundo 'Otro' entre las dos orillas atlánticas: Willa Cather y el sincretismo cultural». Mosaico transatlántico: escritoras, artistas, imaginarios (España-USA, 1830-1940). Coords. Beatriz Ferrús Antón y Alba del Pozo García. Valencia: Publicacions de la Universitat de València, 2015. 85-99.

Alves Lobo, Patrízia. «Todas las voces que me hablan simultáneamente’: Anzaldúa’s Voice from the Borderlands». Estudios ingleses de la Universidad Complutense 23 (2015): 43-54.

BARRANCo UreÑa, EmPar. «Uno de los nuestros: Willa Cather y la guerra». Letras desde la trinchera: testimonios literarios de la Primera Guerra Mundial. Eds. Carme Manuel e Ignacio Ramos. Valencia: Publicacions de la Universitat de València, 2015. 311-22.

Blanco Hidalga, Jesús. «Knowable Conspiracies: Ideology and Form in Jonathan Franzen's The Twenty-Seventh City». Miscelánea 52 (2015): 13-30.

Brito Vera, María Concepción. «A Spatial Reading of Fiona Cheong's Shadow Theatre. The Production of Subversive Female Spaces». English and American Studies in Spain: New Developments and Trends. Eds. Alberto Lázaro Lafuente y María Dolores Porto Requejo. Alcalá de Henares: Servicio de Publicaciones de la Universidad de Alcalá, 2015 (libro electrónico). 69-75.

Bustamante, Fernanda, y Ferrús, Beatriz, eds. Miradas cruzadas: escritoras, artistas e imaginarios (España-EE.UU., 1830-1930). Valencia: Publicacions de la Universitat de València, 2015.

Cajiao CuÉllar, Elsa. «Disentangling Emily Dickinson's Riddles and Encoded Voices in 'My Life Had Stood-a Loaded Gun' and 'I Taste a Liquor Never Brewed'». Atlantis. Journal of the Spanish Association of Anglo-American Studies 37.2 (2015): 27-43.

Calvo García de LeOnaRdo, JuAn José. Traslación, agresión y trasgresión: guerra y sexo ilícito en doce extractos de Hemingway, Mailer, Updike y Nabokov. Valencia: Publicacions de la Universitat de València, 2015.

Casado Carro, María Elena. «The Age-old Trouble: An Analysis of Age and Humor in Beverly Doofrio's Novel Riding in Cars with Boys». ES: Revista de filología inglesa 36 (2015): 87-102.

CAstro Borrego, Silvia. «Integration, Assimilation, and Identity in Lorraine Hansberry's A Raisin in the Sun and Barbara and Carlton Molette's Rosalee Pritchett». Revista canaria de estudios ingleses 70 (2015): 123-42.

Сobo Piñero, María Rocío. «All-Woman Jazz Bands and Gendered Beboppers: Gayl Jones and Gloria Naylor's Jazz Fiction». Revista de estudios norteamericanos 19 (2015): 13-28. 
Cobo Piñero, María Rocío. Sonidos de la diáspora: blues y jazz en Toni Morrison, Alice Walker y Gayl Jones. Sevilla: Arcibel Editores, 2015.

Correoso Ródenas, José Manuel. «Nuevas concepciones espaciales en The Turn of the Screw». Epos: Revista de Filología 31 (2015): 389-404.

Cortés Vieco, Francisco José. «The (Mis)Education of 'the American Girl' in Europe in Anita Loos's Gentlemen Prefer Blondes». Revista de estudios norteamericanos 19 (2015): 29-48.

- «¿Paranoide o paranoia gótica?: del punto de vista de Henry James en The Turn of the Screw hacia múltiples vistas críticas». Analecta Malacitana 39 (2015): 21-43.

Cucarella-Ramon, Vicent. «The Black Female Slave Takes Literary Revenge: Female Gothic Motifs against Slavery in Hannah Crafts's The Bondwoman's Narrative». Journal of English Studies 13 (2015): 19-46.

- «Entre Romanticismo, Antiesclavismo y Espiritualidad: los ecos feministas transculturales de $S a b$ de Gertrudis Gómez de Avellaneda en The Bondwoman's Narrative de Hannah Crafts». RAUDEM. Revista de estudios de las mujeres 3 (2015): 59-86.

DANICA, CĚrcĚ. «Literature as Protest and Solace: The Verse of Alf Taylor». Coolabah 16 (2015): 25-33.

Derrick, Paul Scott. Lines of Thought: 1983-2015. Valencia: Publicacions de la Universitat de València, 2015.

Doménech i Masià, Oreto. Poesía digital: Deena Larsen y Stephanie Strickland. Valencia: Publicacions de la Universitat de València, 2015.

Ibáñez IbáÑEZ, José R. «War and Exile in Aleksandar Hemon's. The Question of Bruno (2000). Verbeia. Revista de estudios filológicos 0 (2015): 204-220.

FERnÁndez-CAPARRós Turina, ANA. «Gertrude Stein y la Gran Guerra: sobre la percepción de los objetos en el frente cotidiano». Letras desde la trinchera: testimonios literarios de la Primera Guerra Mundial. Eds. Carme Manuel e Ignacio Ramos. Valencia: Publicacions de la Universitat de València, 2015. 279-296.

Ferrández San Miguel, María. «The Collusion of Feminist and Postmodernist in E. L. Doctorow's Ragtime». Complutense Journal of English Studies 25 (2015): 97-114.

- «Repurposing 'disreputable genre materials': E.L. Doctorow's Subversion of Hegemonic Gender Configurations in Welcome to Hard Times». Revista de estudios norteamericanos 19 (2015): 67-81.

Ferrús, BeAtriz y del Pozo, Alba, coords. Mosaico transatlántico: escritoras, artistas, imaginarios (España-USA, 1830-1940). Valencia: Publicacions de la Universitat de València, 2015.

Garrido Daneri, Déborah Alejandra. «El holocausto y los recuerdos en The Devil's Arithmetic de Jane Hyatt Yolen». Nerter. Revista dedicada a la literatura, el arte y el conocimiento 25 (2015): 93-101.

Gerke, Amanda. «Walking out on Language: Verbal Spaces in Junot Diaz's Invierno». Revista de estudios norteamericanos 19 (2015): 83-97.

Goicoechea, María, y Salceda, Víctor. «The Mechanic Ear: North American Sound Poetry in the Digital Age». Estudios ingleses de la Universidad Complutense 23 (2015): 129-52.

Gómez Castro, Cristina y Pérez lópez de Heredia, María. «En terreno vedado: género, traducción y censura. El caso de Brokeback Mountain». Traducción y censura: nuevas perspectivas. Eds. Gora Zaragoza Ninet, Juan José Martínez Sierra y José Javier Ávila-Cabrera. Quaderns de Filologia. Estudis Literaris 20 (2015): 35-52.

Gómez López, Jesús IsAíAs. «Isaac Asimov, limericista: la fundación del gróseric». Epos: Revista de Filología 31 (2015): 421-36.

Gomez Moreno, Marta. «La influencia del arquetipo de la gran madre en el bullying a través de Carrie (1974) de Stephen King». Revista del Instituto de Investigación de Estudios de Género de la Universidad de Alicante 25 (2015): 159-182. 
GonZÁlez, Jesús Ángel. «Eastern and Western Promises in Jonathan Franzen's Freedom». Atlantis. Journal of the Spanish Association of Anglo-American Studies 37.2 (2015): 11-29.

GonzÁlez Groba, Constante. «'So far as I and my people are concerned the South is fascist now and always has been': Carson McCullers and the Racial Problem». Atlantis. Journal of the Spanish Association of Anglo-American Studies 37.2 (2015): 63-80.

Gutiérrez Sebastián, RaQuel y Rodríguez Gutiérrez, Borja. «¡Cuán originales y americanos han sido los grandes poetas 'yankees' y a la vez cuán ingleses!: Menéndez Pelayo ante la literatura norteamericana». Mosaico transatlántico: escritoras, artistas, imaginarios (España-USA, 1830-1940). Coords. Beatriz Ferrús Antón y Alba del Pozo García. Valencia: Publicacions de la Universitat de València, 2015. 71-84.

Ibarrola Armendáriz, Aitor. «Sherman Alexie's The Toughest Indian in the World: Amalgamating the Oral and the Written Traditions». Complutense Journal of English Studies 23 (2015): 137-60.

Jin, Ha. Una llegada inesperada y otros relatos. Trad. y Ed. José Ramón Ibáñez Ibáñez y Blasina Cantizano Márquez. Madrid: Encuentro 2015.

Kiczkowski, Adriana. «Identidades y nuevo territorio en Falling Man de Don DeLillo». Identidades en contexto y cultura posmoderna. Ensayos críticos. Eds. Ángel Mateos-Aparicio Martín-Albo y Eduardo de Gregorio-Godeo. Oviedo: KRK Ediciones, 2015. 157-166.

- «World Trade Center: Cause and Target. Kapitoil by Teddy Wayne». English and American Studies in Spain: New Developments and Trends. Eds. Alberto Lázaro Lafuente y María Dolores Porto Requejo. Alcalá de Henares: Servicio de Publicaciones de la Universidad de Alcalá, 2015 (libro electrónico). 106-12.

Lena, Alberto. Alfred Hitchkock, Thornton Wilder y 'La sombra de una duda'. Valladolid: Ediciones de la Universidad de Valladolid, 2015.

Llorens Cubedo, Dídac. «Thought, Sound, Silence, Music: The Turn of the Screw from Henry James to Benjamin Britten». Estudios ingleses de la Universidad Complutense 23 (2015): 97-109.

- «La tierra baldía de T. S. Eliot como poema de posguerra». Letras desde la trinchera: testimonios literarios de la Primera Guerra Mundial. Eds. Carme Manuel e Ignacio Ramos. Valencia: Publicacions de la Universitat de València, 2015. 297-310.

López Cirugeda, Isabel. «Dorothy Parker ante la encrucijada de la Modernidad». Identidades en contexto y cultura posmoderna. Ensayos críticos. Eds. Ángel Mateos-Aparicio Martín-Albo y Eduardo de Gregorio-Godeo. Oviedo: KRK Ediciones, 2015. 73-182.

- «Female Figures of the Jazz Age in Dorothy Parker's Short Stories». The Grove: Working Papers on English Studies 22 (2015): 91-105.

López Peña, Laura. Beyond the Walls: Being with Each Other in Herman Melville's 'Clarel'. Valencia: Publicacions de la Universitat de València, 2015.

López Ramírez, Manuela. «The Haunted House in Toni Morrison's A Mercy». Revista de estudios norteamericanos 19 (2015): 99-113.

- «Gothic Tropes in Toni Morrison's Home: The Scientist-villain Figure and the Maiden in Distress». Revista de filología de la Universidad de La Laguna 33 (2015): 119-132.

— «'Hurt right down the middle... But alive and well': Healing in Toni Morrison's Home». Odisea 16 (2015): 127-142.

- «'What you do to children matters': Motherhood in Toni Morrison's God Help the Child». The Grove: Working Papers on English Studies 22 (2015): 107-19.

Marín Ruiz, Ricardo. «Revisiting Rosinante: Reinterpretations of the Cervantine character in Rosinante to the Road Again, Monsignor Quixote, and Travels with Charley». Epos: Revista de Filología 31 (2015): 437-52.

Marland, Pippa Jane. «The 'Good Step' and Dwelling in Tim Robinson's Stones of Aran: The Advent of 'Psycho-archipelagraphy'». Ecozon@. European Journal of Literature, Culture and Environment 6.1 (2015): 7-24. 
Martínez Pleguezuelos, Antonio J. y GonzÁlez-Iglesias GonZález, Juan David. «La identidad censurada: representación y manipulación de la homosexualidad en la obra Té y simpatía». Traducción y censura: nuevas perspectivas. Eds. Gora Zaragoza Ninet, Juan José Martínez Sierra y José Javier Ávila-Cabrera. Quaderns de Filologia. Estudis Literaris 20 (2015): 53-67.

Mercader Varela, Myriam M. «A Glimpse at Paul Auster and Jorge L. Borges through the Tinted Glass of Quantum Theory». Blue Gum 2 (2015): 43-54.

Merton, Thomas. Diccionario. Trad. William H. Shannon, Christine M. Bochen y Patrick F. O'Connell. Bilbao: Mensajero, 2015.

Monterrey, Tomás. «'Painfully brilliant sunrise. The mountain is silent': Nature, Transgression and Justice in the Opera Brokeback Mountain». English and American Studies in Spain: New Developments and Trends. Eds. Alberto Lázaro Lafuente y María Dolores Porto Requejo. Alcalá de Henares: Servicio de Publicaciones de la Universidad de Alcalá, 2015 (libro electrónico). 121-128.

Morales Jareño, Isabel. «Joanna Russ's The Female Man: Claiming Feminism and Sexuality across Utopian Communities». Verbeia. Revista de estudios filológicos 0 (2015): 221-234.

Nalerio, Juliana. «The Patriarch's Balls: Class-consciousness, Violence, and Dystopia in George Saunders' Vision of Contemporary America». Miscelánea 52 (2015): 89-102.

Niewiadomska-Flis, Ursula y Budzynska, Magdalena. «The Clash Between Memory and the Self in Walker Percy's The Last Gentleman and The Second Coming». Atlantis. Journal of the Spanish Association of Anglo-American Studies 37.2 (2015): 81-98.

Ortells Montón, Elena. «Hybrid Subjects and Fluid Identities in Women's Indian Captivity Narratives: The Stories of Frances Slocum and Olive Oatman». Revista de estudios norteamericanos 19 (2015): 133-50.

PÁrRaga, JaVier Martín. «Kurt Vonnegut's Dual Roots». Revista canaria de estudios ingleses 70 (2015): 143-154.

PeÑalba García, Mercedes. "Writing the Self, Drawing the Self: Identity and Self-Reflexivity in Craig Thompson's Graphic Memoir Blankets». Culture, Language and Representation 14 (2015): 155-180.

Pérez Galván, Paola. «El modernismo de Kay Boyle a través de algunos relatos». Nerter: Revista dedicada a la literatura, el arte y el conocimiento 25 (2015): 102-109.

Pérez García, Ana Belén. «Female Native American Storytelling: Female Storytellers in Native culture. Presence in Contemporary Native American Literature. Leslie Marmon Silko». The Grove: Working Papers on English Studies 22 (2015): 121-133.

Pérez Villalón, Óscar. «El montaje y el gesto (Ezra Pound / Henri Michaux: dos poéticas del ideograma». $452^{\circ}$. Revista de teoría de la literatura y literatura comparada 13 (2015): 99-114.

Piñero Gil, Eulalia. «Music Evoked Pictures in Her Mind: Sensory and Artistic Synaesthesia in Kate Chopin's The Awakening». Estudios Ingleses de la Universidad Complutense 23 (2015): 111-127.

Piqueras, Belén. «Texts As Bodies/Bodies As Texts: The Barthes-Gass Connection». Babel afial: Aspectos de filología inglesa y alemana 24 (2015): 55-72.

Piqueras Fraile, María Del Rosario. «La identidad sureña a través de la obra de Joel Chandler Harris». Identidades en contexto y cultura posmoderna. Ensayos críticos. Eds. Ángel MateosAparicio Martín-Albo y Eduardo de Gregorio-Godeo. Oviedo: KRK Ediciones, 2015. 95-104.

PRIETo García-CAÑEdo, SARA. «La paradoja del reportero de guerra: romanticismo y decadencia en With the Allies de Richard Harding Davis». Letras desde la trinchera: testimonios literarios de la Primera Guerra Mundial. Eds. Carme Manuel e Ignacio Ramos. Valencia: Publicacions de la Universitat de València, 2015. 359-74.

Rodríguez Guerrero-Strachan, Santiago. «Poéticas pictóricas. A propósito de los ensayos de John Ashbery y José Ángel Valente y el expresionismo abstracto». Identidades en contexto y cultura posmoderna. Ensayos críticos. Eds. Ángel Mateos-Aparicio Martín-Albo y Eduardo de Gregorio-Godeo. Oviedo: KRK Ediciones, 2015. 127-137. 
Tydal, FredriK. «Towards a Unity of Dos Passos's and Hemingway's Aesthetics in The Spanish Earth». The Grove: Working Papers on English Studies 22 (2015): 205-12.

Wellman, Donald. Remando de noche. Trad. Francisca González Arias, ed. Nicolás Estévez. Valencia: Publicacions de la Universitat de València, 2015.

Zabalgoitia, Mauricio. «Las estadounidenses visitan España: la literatura de viajes entre el testimonio y la mercancía». Mosaico transatlántico: escritoras, artistas, imaginarios (EspañaUSA, 1830-1940). Coords. Beatriz Ferrús Antón y Alba del Pozo García. Valencia: Publicacions de la Universitat de València, 2015. 39-49.

Zamorano, Ana Isabel. «Los límites del cuerpo 'queer': sombras transparentes en Fun Home de Alison Bechdel». Nerter: Revista dedicada a la literatura, el arte y el conocimiento 25 (2015): $36-42$. 


\title{
C. OTRAS LITERATURAS EN LENGUA INGLESA
}

\author{
Mariángel SolÁns García \\ UNED \\ masolans@madrid.uned.es \\ Dídac Llorens Cubedo \\ UNED \\ dllorens@flog.uned.es
}

\section{ESTUDIOS SOBRE LITERATURA AFRICANA}

Álvarez Sánchez, Patricia. «J. M. Coetzee's In the Heart of the Country: Is Magda a New Don Quixote?». English and American Studies in Spain: New Developments and Trends. Eds. Alberto Lázaro Lafuente y María Dolores Porto Requejo. Alcalá de Henares: Servicio de Publicaciones de la Universidad de Alcalá, 2015 (libro electrónico). 60-68.

Gil Navaeira, Isabel. «La auto-reafirmación de la identidad femenina africana en la novela Kehinde, de Buchi Emecheta». Identidades en contexto y cultura posmoderna. Ensayos críticos. Eds. Ángel Mateos-Aparicio Martín-Albo y Eduardo de Gregorio-Godeo. Oviedo: KRK Ediciones, 2015. 69-75.

MARTín-RUiz, SARA. «'The way the Irish asylum system turns people into un-human is my problem': An Interview with Ifedinma Dimbo». Estudios irlandeses. Journal of Irish Studies 10 (2015): 109-114.

MichтA, KamiL. «Framing Humanity by Framing Nature: John Maxwell Coetzee's Disgrace in the Context of Kant's Theory of Morality». Ecozon@. European Journal of Literature, Culture and Environment 6.1 (2015): 137-150.

Stefanova Radoulska, Svetlana. "'Positioning is, above all, a matter of representation': J. M. Coetzee's and the Transformative Power of Transgression». Cultura, lenguaje y representación 14 (2015): 181-200.

\section{ESTUDIOS SOBRE LITERATURA INDIA/ASIÁTICA}

Alonso Breto, IsABel. «Water, White Tigers and Corrupt Neoliberalism: Controversial Entrepreneurs in Recent Fiction from the Subcontinent». Indialogs. Spanish Journal of India Studies 2 (2015): 5-22.

Athanasiades, Andreas. «Reposessing Islam: Affective Identity and Islamic Fundamentalism in Hanif Kureishi». Indialogs. Spanish Journal of India Studies 2 (2015): 55-71.

Brito Vera, María Concepción. «A Spatial Reading of Fiona Cheong's Shadow Theatre. The Production of Subversive Female Spaces». English and American Studies in Spain: New Developments and Trends. Eds. Alberto Lázaro Lafuente y María Dolores Porto Requejo. Alcalá de Henares: Servicio de Publicaciones de la Universidad de Alcalá, 2015 (libro electrónico). 69-75. González, María Luz, y Oliva, Juan Ignacio. «Bharati Mukherjee's Struggle Against Cultural Balkanization: the Forging of a New American Immigrant Writing». Indialogs. Spanish Journal of India Studies 2 (2015): 72-92.

Goonetileke, D.C.R.A. «Salman Rushdie's Midnight's Children, The Play as Text and Performance: An Introductory Note». Indialogs. Spanish Journal of India Studies 2 (2015): 119-123. 
Hand, Felicity. «Coping with Khandaanity in Diaspora Spaces: South Asian Women in East Africa». Revista canaria de Estudios Ingleses 70 (2015): 13-40.

— «A Talk with Siddarth Dhanvant Shanghvi». Indialogs. Spanish Journal of India Studies 2 (2015): 133-137.

Hanrahan, Fiedhlim. «The Poverty Tour: Life in the Slums of Mumbai and Manila as Seen in Danny Boyle's Slumdog Millionaire and Merlinda Bobis's The Solemn Lantern Maker». Atlantis. Journal of the Spanish Association of Anglo-American Studies 37.1 (2015): 101-119.

Navarro Tejero, Antonia. «Indianness, Hindu Nationalism and Authenticity: United Forward Women, Capable India». Revista canaria de Estudios Ingleses 70 (2015): 67-88.

O'CONNOR, MAURICE. «South Asian Muslim Identity in the UK». Revista canaria de Estudios Ingleses 70 (2015): 41-66.

Wallhead Salway, Celia Margaret. «Meditations on Genre in Salman Rushdie's Joseph Anton». Revista canaria de Estudios Ingleses 70 (2015): 89-104.

\section{ESTUDIOS SOBRE LITERATURA AUSTRALIANA Y NEOCELANDESA}

Hanrahan, Fiedhlim. «The Poverty Tour: Life in the Slums of Mumbai and Manila as Seen in Danny Boyle's Slumdog Millionaire and Merlinda Bobis's The Solemn Lantern Maker». Atlantis. Journal of the Spanish Association of Anglo-American Studies 37.1 (2015): 101-119.

Hawryluk Lynda, Shilton Leni. «Negotiating 'Negative Capability': The Role of Place in Writing». Coolabah 16 (2015): 48-73.

Holden Rønning, AnNe. «Louisa Lawson and the Woman Question». Coolabah 16 (2015): 74-86.

RAHBEK, Ulla. «Developing a Connective Feminine Discourse: Drusilla Modjeska on Women's Lives, Love and Art». Coolabah 16 (2015): 101-111.

Ribas Segura, Catalina. «Identity and Friendship in Hsu-Ming Teo's Behind the Moon (2000)». Coolabah 16 (2015): 112-121.

\section{ESTUDIOS SOBRE LITERATURA CANADIENSE}

Alonso Breto, Isabel. «Althea Prince's Loving This Man: An Intersectional Approach to Migration, Gender and Race Politics». ES: Revista de filología inglesa 36 (2015): 27-46.

Carmona-Rodríguez, Pedro-Miguel. «Neither Chuckwagons, nor Saskatoons, and a Missing Malboro Man: Postcolonialism, Regionalism and the Ineffable Canadian West». Miscelánea 52 (2015): 31-49.

Casado Gual, Núria. «Joanna McClelland Glass' Mrs. Dexter and Her Daily and the Inter-play Between Race, Gender and Class». Canada and Beyond: A Journal of Canadian Literary and Cultural Studies 5 (2015): 1-26.

Cucarella-Ramón, Vicent. «Liminality and (Trans)nationalism in the Rethinking of the African Canadian Subjectivity: Esi Edugyan's The Second Life of Simon Tyne». Canada and Beyond: A Journal of Canadian Literary and Cultural Studies 5 (2015): 27-50.

Moure, ErIN. «Writing in Secession: Biopoetics and the Galician Imaginaire of Chus Pato in Canada». Canada and Beyond: A Journal of Canadian Literary and Cultural Studies 5 (2015): 72-87.

Pich Ponce, Eva. «La literatura canadiense y la Primera Guerra Mundial». Letras desde la trinchera: testimonios literarios de la Primera Guerra Mundial. Eds. Carme Manuel e Ignacio Ramos. Valencia: Publicacions de la Universitat de València, 2015. 375-386.

Rodríguez Nieto, Natalia. «'Bring What You Know of Life to Life: Alice Munro's Storytelling». Verbeia. Revista de estudios filológicos 0 (2015): 264-274.

Roupakia, Lydya Efthymia. «'Art-iculating' Affective Citizenship: Dionne Brand's What We All Long For». Atlantis. Journal of the Spanish Association of Anglo-American Studies 37.1 (2015): 31-50. 


\title{
D. ENFOQUES TEÓRICOS O GENERALISTAS
}

\author{
Dídac Llorens Cubedo \\ UNED \\ dllorens@ flog.uned.es \\ MARIÁNGEl Soláns García \\ UNED \\ masolans@madrid.uned.es
}

Alonso Recarte, Claudia. «De caballos, perros y palomas. El animal en la cultura militar de la Primera Guerra Mundial y su reflejo en la literatura». Letras desde la trinchera: testimonios literarios de la Primera Guerra Mundial. Eds. Carme Manuel e Ignacio Ramos. Valencia: Publicacions de la Universitat de València, 2015. 387-403.

Escudero Pérez, Jimena, y Moreno Álvarez, Alejandra. «Cíborgs: cuerpo, identidad, metáfora y su utilización literaria». Identidades en contexto y cultura posmoderna. Ensayos críticos. Eds. Ángel Mateos-Aparicio Martín-Albo y Eduardo de Gregorio-Godeo. Oviedo: KRK Ediciones, 2015. 183-191.

GONZÁLEZ FISAC, JeSÚs. «¿Quién teme a los estudios culturales? La interdisciplinariedad o el problema de la identidad en Judith Butler». Identidades en contexto y cultura posmoderna. Ensayos críticos. Eds. Ángel Mateos-Aparicio Martín-Albo y Eduardo de Gregorio-Godeo. Oviedo: KRK Ediciones, 2015. 21-30.

Herrero Ruiz, Javier. «At the Crossroads between Literature, Culture, Linguistics, and Cognition: Local Character-based Metaphors in Fairy Tales». Journal of English Studies 13 (2015): 47-70.

Manuel, Carme e Ignacio Ramos, eds. Letras desde la trinchera: testimonios literarios de la Primera Guerra Mundial. Valencia: Publicacions de la Universitat de València, 2015.

Martín-Alegre, Sara, ed. Gender and Feminism: The Student's View. Barcelona: Universitat Autònoma de Barcelona, 2015 (libro electrónico).

Martínez Serrano, Leonor María. «The Power and Promise of 21st Literary Criticism». Odisea 16 (2015): 179-196.

Mateos-Aparicio Martín-Albo, Ángel y De Gregorio-Godeo, Eduardo, eds. Identidades en contexto y cultura posmoderna. Ensayos críticos. Oviedo: KRK Ediciones, 2015.

Mérida Jiménez, Rafael Manuel. «Vindicaciones para teorías». Nerter. Revista dedicada a la literatura, el arte y el conocimiento 25 (2015): 13-18.

Peralta, Jorge Luis. «Escrituras disidentes: algunas propuestas teóricas». Nerter. Revista dedicada a la literatura, el arte y el conocimiento 25 (2015): 19-27.

Ramos Arteaga, José Antonio. «La teoría que vendrá». Nerter. Revista dedicada a la literatura, el arte y el conocimiento 25 (2015): 9-12.

RODRÍGUeZ, AlEXANDRA. «La teoría 'queer' y su labor de transformación social en los cuerpos trans». Nerter. Revista dedicada a la literatura, el arte y el conocimiento 25 (2015): 54-58.

Ruiz Callejón, Encarnación. «Qasim Amin y John Stuart Mill: las razones de la esclavitud femenina». Feminismos 26 (2015): 57-81.

Seguí Aznar, Juana María, y Bastida Rodríguez, Patricia. Entre la calma y la inspiración: Diccionario de autores anglófonos en Baleares. Palma de Mallorca: José J. de Olañeta Editor, 2015.

Sмyth, Ailbhe. «Telling the Truth About Women's Lives». Estudios irlandeses. Journal of Irish Studies 10 (2015): 115-119. 


\title{
E. ESTUDIOS DE TRADUCCIÓN
}

\author{
José JAVIER ÁvILA-CABRERA \\ Universidad Complutense de Madrid \\ josejaav@ucm.es
}

Abril Martí, María Isabel. «La interpretación en contextos de violencia de género con referencia al caso español». TRANS (Revista de Traductología) 19.1 (2015): 77-94.

Aguayo Arrabal, Natividad y Ramírez Delgado, Cristina. «El traductor-intérprete, ¿presente en el comercio exterior?». Investigación emergente en Traducción e Interpretación. Eds. Raquel Lázaro Gutiérrez, María del Mar Sánchez Ramos y Francisco Javier Vigier Moreno. Granada: Editorial Comares, 2015. 11-24.

Aguilera Crespillo, María de la Presentación, y Corpas Pastor, Gloria. «La constitución de sociedades offshore británicas: aspectos jurídicos y consecuencias para la traducción jurada al español». Traducimos desde el sur. VI Congreso Internacional de la AIETI. Ed. José Jorge Amigo Extremera. Las Palmas de Gran Canaria: Universidad de Las Palmas de Gran Canaria, 2015. 655-672.

Aguirre Fernández-Bravo, Elena, y Roca Urgorri, Ana María. «Perfiles y roles del intérprete en los servicios públicos: el intérprete mediador como modelo de compromiso». Investigación emergente en Traducción e Interpretación. Eds. Raquel Lázaro Gutiérrez, María del Mar Sánchez Ramos y Francisco Javier Vigier Moreno. Granada: Editorial Comares, 2015. 19-34.

Alcalde PeÑalver, Elena. «Estudio empírico de la profesión del traductor financiero en España». ENTRECULTURAS 7-8 (2015): 281-292.

- «¿Qué es la traducción financiera? Limitación del concepto mediante la aplicación de diversos parámetros?». Estudios de Traducción 5 (2015): 119-131.

Alcina Caudet, María Amparo. «Estándares y formatos de intercambio en terminología». Revista Tradumàtica 13 (2015): 571-583.

Allen, JefF. «Translation Standards: Which One?». Revista Tradumàtica 13 (2015): 545-551.

Alonso Almeida, Francisco, y Carrió Pastor, María Luisa. «Una aproximación a la traducción de seem al español». Traducimos desde el sur. VI Congreso Internacional de la AIETI. Eds. José Jorge Amigo Extremera. Las Palmas de Gran Canaria: Universidad de Las Palmas de Gran Canaria, 2015. 393-403.

Alonso Araguás, Icíar, Páez Rodríguez, Albe y Samaniego Sastre, Mario, eds. Traducción y representaciones del conflicto desde España y América. Salamanca y Temuco: Universidad de Salamanca y Ediciones Universidad Católica de Temuco, 2015.

Alonso Jiménez, Elisa. «Francisco Ayala and his Professional Approach to Translation: Theory and Practice». TRANS (Revista de Traductología) 19.2 (2015): 195-209.

Alonso Veloso, María José. «La recepción europea del Marco Bruto de Quevedo: traducciones hasta el siglo XVIII». La transmisión de la obra de Quevedo: edición, recepción, traducción. Coords. M. Á. Candelas y Flavia Gherardi. Vigo: Editorial Academia del Hispanismo, 2015. 29-65.

Alpuente Civera, Miguel, Martínez Vilinsky, Bárbara, Marco Borillo, Josep, y Oltra Ripoll, MARía D. «Propuesta de actividades de escritura creativa para las clases de traducción literaria». Hermeneus 17 (2015): 31-60.

Álvarez De Morales Mercado, Cristina. «El subtitulado para personas sordas como discurso narratológico». Sendebar 26 (2015): 57-81. 
Amigo Extremera, José Jorge, ed. Traducimos desde el sur. VI Congreso Internacional de la AIETI. Las Palmas de Gran Canaria: Universidad de Las Palmas de Gran Canaria, 2015.

ANFuso, MATTEO. «Ambigüedad y direccionalidad en interpretación simultánea y traducción (semi) a la vista». Investigación emergente en Traducción e Interpretación. Eds. Raquel Lázaro Gutiérrez, María del Mar Sánchez Ramos y Francisco Javier Vigier Moreno. Granada: Editorial Comares, 2015.

Angelelli, Claudia. «Justice for All? Issues Faced by Linguistic Minorities and Border Patrol Agents during Interpreted Arraignment Interviews». Legal Interpreting at a Turning Point. Eds. María Jesús Blasco Mayor y Maribel del Pozo Triviño. MonTI 7 (2015): 181-205.

Araújo, Silvia y Correia, Ana. «Corpus y formación de intérpretes: un estudio exploratorio». Metodologías y aplicaciones en la investigación en traducción e interpretación con corpus. The Hermeneus Conference. Eds. María Teresa Sánchez Nieto, Susana Álvarez Álvarez; Verónica Arnáiz-Uzquiza, Teresa Ortego Antón, Leticia Santamaría Ciordia y Rosa Fernández Muñiz. Valladolid: Universidad de Valladolid, 2015. 165-181.

Arnáiz Uzquiza, Verónica, Álvarez Álvarez, Susana y Corell Almuzara, Alfredo. «Corpus de textos audiovisuales frente a corpus de textos escritos. La traducción de micropíldoras de aprendizaje». Metodologías y aplicaciones en la investigación en traducción e interpretación con corpus. The Hermeneus Conference. Eds. María Teresa Sánchez Nieto, Susana Álvarez Álvarez; Verónica Arnáiz-Uzquiza, Teresa Ortego Antón, Leticia Santamaría Ciordia y Rosa Fernández Muñiz. Valladolid: Universidad de Valladolid, 2015. 237-250.

Ávila-Cabrera, José Javier. «An Account of the Subtitling of Offensive and Taboo Language in Tarantino's Screenplays». Sendebar 26 (2015): 37-56.

— «Propuesta de modelo de análisis del lenguaje ofensivo y tabú en la subtitulación». VERBEIA. Revista de estudios filológicos 0 (2015): 8-27.

- «Subtitling Tarantino's Offensive and Taboo Dialogue Exchanges into European Spanish: The Case of Pulp Fiction». Revista de Lingüística y Lenguas Aplicadas 10 (2015): 1-11.

BAigorri Jalón, Jesús y Russo, Mariachiara, coords. La interpretación en entornos judiciales. TRANS (Revista de Traductología) 19.1 (2015): 1-152.

BAÑos, Rocío. «Los estudios descriptivos de traducción en la investigación de la traducción para el doblaje». Traducción, ideología y poder en la ficción audiovisual. Eds. Frederic Chaume y Mabel Richart Marset. Prosopopeya. Revista de crítica contemporánea 9 (2014-2015): 27-52.

Barbato, Lucia. «Estudios de evaluación de la calidad de la interpretación». Transfer. Revista electrónica sobre traducción e interculturalidad 10.1 (2015): 91-106.

BARTOLl TeIXIDOR, EDUARD. Introducción a la traducción audiovisual. Barcelona: Editorial UOC, 2015.

Basich Peralta, Kora Evangelina. «La enseñanza de la traducción en la frontera norte de México: condiciones y situaciones que inciden en su implementación». Traducimos desde el sur. VI Congreso Internacional de la AIETI. Ed. José Jorge Amigo Extremera. Las Palmas de Gran Canaria: Universidad de Las Palmas de Gran Canaria, 2015. 141-152.

Benítez Castro, Beatriz. «Código de buenas prácticas entre ACE Traductores y la Unión de Correctores». Vasos comunicantes 46 (2015): 33-36.

Bestué Salinas, Carmen. «El deber de información como criterio de aceptabilidad de las traducciones jurídicas de las páginas web». TRANS (Revista de Traductología) 19.2 (2015): 22524I.

Blasco Mayor, María Jesús y del Pozo Triviño, Maribel. «La interpretación judicial en España en un momento de cambio». Legal Interpreting at a Turning Point. Eds. María Jesús Blasco Mayor y Maribel del Pozo Triviño. MonTI 7 (2015): 9-71.

Borrás, Manuel, Brouwer, Ronald, Jaume, Andreu, Rod, Carlos, y de Miguel Crespo, Olivia. «Mesa redonda. Traducir poesía y teatro, editar poesía y teatro». Vasos comunicantes 46 (2015): 75-92. 
Bosch Benítez, Amalia y Aranda, Lucía. «Kipling: la traducción de la identidad». Traducimos desde el sur. VI Congreso Internacional de la AIETI. Eds. José Jorge Amigo Extremera. Las Palmas de Gran Canaria: Universidad de Las Palmas de Gran Canaria, 2015. 229-244.

Campos, José Aníbal. «Traducir poesía: en la cópula de las ideas». Vasos comunicantes 46 (2015): $19-22$

Carlucci, Laura y Álvarez de Morales, Cristina, eds. Insights into Multimodal Translation and Accessibility. Láchar (Granada): Tragacanto, 2015.

Carrera Fernández, Judith y Ramiro Valderrama, Manuel. «¿A qué español traducimos? Delimitación del concepto 'español estándar' aplicado a la traducción». Traducimos desde el sur. VI Congreso Internacional de la AIETI. Eds. José Jorge Amigo Extremera. Las Palmas de Gran Canaria: Universidad de Las Palmas de Gran Canaria, 2015. 366-379.

Carrió Pastor, María Luisa. «Mapping the Cultural Interference of Term Variation». CLINA 1.1 (2015): 29-45.

Casamayor Maspóns, Reynaldo. «La sustanciación lingüística del procedimiento penal: el complemento traslativo (I)». ENTRECULTURAS 7-8 (2015): 497-520.

- «La sustanciación lingüística del procedimiento penal: el complemento traslativo (II)». ENTRECULTURAS 7-8 (2015): 521-541.

Castellano Martínez, José María. «Introducción a la traducción de textos ECOFIN de la UE: marcos teóricos, agentes y casos prácticos». Hikma 14 (2015): 75-93.

Cerezo Herrero, Enrique. «English Language Teaching for Translator and Interpreter Trainees: Syllabus Analysis and Design». Quaderns. Revista de Traducció 22 (2015): 289-306.

Cerezo Merchán, Beatriz y Vulpoiu, Laura. «La traducción audiovisual en Rumanía». Linguae. Revista de la Sociedad Española de Lenguas Modernas 2 (2015): 123-159.

CÉSAR DEL AMO, IrIs. «Análisis de la traducción del videojuego To the Moon por parte de fans». ENTRECULTURAS 7-8 (2015): 697-717.

Chaume, Frederic y Richart Marset, Mabel, eds. Traducción, ideología y poder en la ficción audiovisual. Prosopopeya. Revista de crítica contemporánea 9 (2014-2015): 1-330.

- «Del paradigma descriptivista al giro cultural en traducción audiovisual». Traducción, ideología y poder en la ficción audiovisual. Eds. Frederic Chaume y Mabel Richart Marset. Prosopopeya. Revista de crítica contemporánea 9 (2014-2015): 17-26.

Chico Rico, Francisco. «La traducción del texto político: características pragmático-discursivas y estrategias traductológicas». Tonos Digital. Revista Electrónica de Estudios Filológicos 29 (2015).

Corredor Plaja, ANNA-Maria. «Llegir, interpretar, traduir: el difícil equilibri entre el text original i el text meta». Quaderns. Revista de Traducció 22 (2015): 233-243.

Corsellis, ANN. «Strategies for Progress: Looking for Firm Ground». Legal Interpreting at a Turning Point. Eds. María Jesús Blasco Mayor y Maribel del Pozo Triviño. MonTI 7 (2015): 101-114.

Costa, Beverley. «Working as a Team: The Importance of Training and Clinical Supervision of Interpreters and Practitioners for Best Practice in Gender Violence Contexts». Construir puentes de comunicación en el ámbito de la violencia de género. I Congreso Internacional SOS-VICS. Eds. Maribel del Pozo Triviño, Carmen Toledano Buendía, David Casado-Neira y Doris Fernandes del Pozo. Granada: Comares, 2015. 61-72.

Crezee, Ineke Hendrika Martine, Burn, Jo Anna y Gailani, Nidar. «Authentic Audiovisual Resources to Actualise Legal Interpreting Education». Legal Interpreting at a Turning Point. Eds. María Jesús Blasco Mayor y Maribel del Pozo Triviño. MonTI 7 (2015): 271-293.

Dasilva Fernández, Xosé Manuel. «La opacidad de la autotraducción entre lenguas asimétricas». TRANS (Revista de Traductología) 19.2 (2015): 171-182.

De marco, Marcella. «Enlazando (estudios) de género y traducción (audiovisual)». Traducción, ideología y poder en la ficción audiovisual. Eds. Frederic Chaume y Mabel Richart Marset. Prosopopeya. Revista de crítica contemporánea 9 (2014-2015): 99-110. 
Di Giovanni, Elena. «Pinocho para todos: La combinación de la audiodescripción con la escritura de guiones». Traducción, ideología y poder en la ficción audiovisual. Eds. Frederic Chaume y Mabel Richart Marset. Prosopopeya. Revista de crítica contemporánea 9 (2014-2015): 193-218.

Díaz-CinTas, Jorge. «Multilingüismo, traducción audiovisual y estereotipos: el caso de Vicky Cristina Barcelona». Traducción, ideología y poder en la ficción audiovisual. Eds. Frederic Chaume y Mabel Richart Marset. Prosopopeya. Revista de crítica contemporánea 9 (2014-2015): 135-162.

Durán Muñoz, Isabel, Corpas Pastor, Gloria, Ha, Le An, y Mitkov, Ruslan. «Introducing ProTermino: A New Tool Aimed at Translators and Terminologists». Traducimos desde el sur. VI Congreso Internacional de la AIETI. Eds. José Jorge Amigo Extremera. Las Palmas de Gran Canaria: Universidad de Las Palmas de Gran Canaria, 2015. 623-638.

ERrico, Elena. «La calidad en interpretación: Estado de la cuestión y perspectivas de investigación». Tonos Digital. Revista Electrónica de Estudios Filológicos 29 (2015).

Errico, Elena y Morelli, Mara. «La palabra a los oyentes: los comentarios del público en un cuestionario sobre la percepción de la calidad de la interpretación consecutiva de estudiantes en prácticas». Insights in Interpreting. Status and Developments. Eds. Catalina Iliescu Gheorghiu y Juan Miguel Ortega Herráez. MonTI Special Issue 2 (2015): 281-301.

FAYA OrNia, GoRETTI. «La necesidad de servicios de traducción e interpretación en el sector sanitario. La situación en Londres, Düsseldorf y Madrid». ENTRECULTURAS 7-8 (2015): 543-574.

- «Estudio contrastivo del folleto médico (inglés-español)». Quaderns. Revista de Traducció 22 (2015): 347-362.

Fernández Gil, María Jesús. «Censura 2.0 y Holocausto: el control de contenidos y su impacto en la imagen transnacional del genocidio nazi». Traducción y censura: nuevas perspectivas. Eds. Gora Zaragoza Ninet, Juan José Martínez Sierra y José Javier Ávila-Cabrera. Quaderns de Filologia. Estudis Literaris 20 (2015): 201-217.

Fernández GonZÁlez, Vicente. «Las mil maneras de traducir poesía». Vasos comunicantes 46 (2015): 39-53.

FERnÁNDEZ Martínez, CRISTINA. «Homogeneización cultural, transferencia y diferenciación cultural en la terminología del mundo de la moda inglés-francés-español. Implicaciones para la traducción editorial de textos de moda». ENTRECULTURAS 7-8 (2015): 307-343.

Fernández Pérez, María Magdalena. «Propuestas de ejercicios de simulación para la didáctica de la interpretación telefónica». Insights in Interpreting. Status and Developments. Eds. Catalina Iliescu Gheorghiu y Juan Miguel Ortega Herráez. MonTI Special Issue 2 (2015): 259-279.

FranCo AiXelá, JAVIER. «Bibliografía especializada sobre traducción e interpretación: la revista MonTI (universidades de Alicante, Jaume I y València) (2009-2015)». ENTRECULTURAS 7-8 (2015): 853-870.

— «La traducción de textos científicos y técnicos». Tonos Digital. Revista Electrónica de Estudios Filológicos 29 (2015).

García Bértoa, Emma y García Sanz, Santiago. «Videojuegos y normalización lingüística: localización de Maniac Mansion al gallego». Traducimos desde el sur. VI Congreso Internacional de la AIETI. Ed. José Jorge Amigo Extremera. Las Palmas de Gran Canaria: Universidad de Las Palmas de Gran Canaria, 2015. 476-486.

García Izquierdo, Isabel. «La competencia en lengua materna (español) de los estudiantes de traducción e interpretación: Un estudio de caso». Hermeneus 17 (2015): 87-100.

García Morales, Goretti. «La importancia del texto para la didáctica de la interpretación». Traducimos desde el sur. VI Congreso Internacional de la AIETI. Ed. José Jorge Amigo Extremera. Las Palmas de Gran Canaria: Universidad de Las Palmas de Gran Canaria, 2015. 94-104.

García Oya, Elisabet. «Interpretación de conferencias e ideología: resultados de un experimento en interpretación simultánea». Traducimos desde el sur. VI Congreso Internacional de la AIETI. Ed. José Jorge Amigo Extremera. Las Palmas de Gran Canaria: Universidad de Las Palmas de Gran Canaria, 2015. 53-74. 
Gerding Salas, Constanza y Díaz Castro, Cristian. «Hacia una propuesta socioconstructivista para el aprendizaje de la traducción». ENTRECULTURAS 7-8 (2015): 151-178.

Gómez Castro, Cristina y Pérez López de Heredia, María. «En terreno vedado: género, traducción y censura. El caso de Brokeback Mountain». Traducción y censura: nuevas perspectivas. Eds. Gora Zaragoza Ninet, Juan José Martínez Sierra y José Javier Ávila-Cabrera. Quaderns de Filologia. Estudis Literaris 20 (2015): 35-52.

GonzÁlez Rodríguez, María Jesús. «Interpretar escuchas telefónicas en ámbito judicial: análisis descriptivo y metodología operacional». La interpretación en entornos judiciales. Coord. Jesús Baigorri Jalón y Mariachiara Russo. TRANS (Revista de Traductología) 19.1 (2015): 109-129.

González Vera, Pilar. «The Cultural Dimension in Shrek and Shrek Forever After». Traducimos desde el sur. VI Congreso Internacional de la AIETI. Ed. José Jorge Amigo Extremera. Las Palmas de Gran Canaria: Universidad de Las Palmas de Gran Canaria, 2015. 487-506.

GóRSKA, KATARZYNA. «Evidencias y contraintuiciones en la traducción indirecta audiovisual. El caso particular del documental Komeda. La banda sonora d'una vida». Transfer. Revista electrónica sobre traducción e interculturalidad 10.1-2 (2015): 107-125.

GRANELl ZAFRA, JOAQUín. «La evaluación de la investigación: criterios de evaluación en Humanidades y el caso de la Traducción e Interpretación». Investigación Bibliotecológica 29.66 (2015): 57-78.

Gutiérrez Lanza, Camino. «Retranslation and Counterculture in Post-Francoist and Modern-day Spain: Woody Guthrie's Bound for Glory in Star Books (1977) and Global Rhythm Press (2009)». Traducción y censura: nuevas perspectivas. Eds. Gora Zaragoza Ninet, Juan José Martínez Sierra y José Javier Ávila-Cabrera. Quaderns de Filologia. Estudis Literaris 20 (2015): 125-144.

Hale, Sandra Beatriz. «Approaching the Bench: Teaching Magistrates and Judges how to Work Effectively with Interpreters». Legal Interpreting at a Turning Point. Eds. María Jesús Blasco Mayor y Maribel del Pozo Triviño. MonTI 7 (2015): 163-180.

Handi, Elhassane Benhaddou. «La posibilidad de una comunicación de calidad con las mujeres extranjeras víctimas de violencia». Construir puentes de comunicación en el ámbito de la violencia de género. I Congreso Internacional SOS-VICS. Eds. Maribel del Pozo Triviño, Carmen Toledano Buendía, David Casado-Neira y Doris Fernandes del Pozo. Granada: Comares, 2015. 55-60.

Hart Robertson, Margaret. «Identity in Transit: Interpreting Med Voices». Traducimos desde el sur. VI Congreso Internacional de la AIETI. Ed. José Jorge Amigo Extremera. Las Palmas de Gran Canaria: Universidad de Las Palmas de Gran Canaria, 2015. 23-25.

Hernández Guerrero, María José. «La traducción en The Huffington Post». Hermeneus 17 (2015): 111-136. Hertog, EriK. «The Right of Victims to Understand and to be Understood: The SOS-VICS Project Against its EU Background». Construir puentes de comunicación en el ámbito de la violencia de género. I Congreso Internacional SOS-VICS. Eds. Maribel del Pozo Triviño, Carmen Toledano Buendía, David Casado-Neira y Doris Fernandes del Pozo. Granada: Comares, 2015. 19-36.

— «Looking Back While Going Forward: 15 Years of Legal Interpreting in the EU». La interpretación en entornos judiciales. Coord. Jesús Baigorri Jalón y Mariachiara Russo. TRANS (Revista de Traductología) 19.1 (2015): 15-31.

- «Directive 2010/64/EU of the European Parliament and of the Council on the Right to Interpretation and Translation in Criminal Proceedings: Transposition Strategies with Regard to Interpretation and Translation». Legal Interpreting at a Turning Point. Eds. María Jesús Blasco Mayor y Maribel del Pozo Triviño. MonTI 7 (2015): 73-100.

Huertas, Cristina A., y Burgos Bárcena, Ana. «La traducción de los culturemas en el doblaje y la subtitulación de Mad Men». Cultura, lenguaje y representación 14 (2015): 61-123.

Iliescu Gheorghiu, Catalina y Ortega Herráez, Juan Miguel. «El intérprete oye voces... perspectivas académicas y profesionales radiografiadas y anotadas». Insights in Interpreting. Status and Developments. Eds. Catalina Iliescu Gheorghiu y Juan Miguel Ortega Herráez. MonTI Special Issue 2 (2015): 9-36. 
Iliescu Gheorghiu, Catalina y Ortega Herráez, Juan Miguel, Eds. Insights in Interpreting. Status and Developments. MonTI Special Issue 2 (2015): 1-308.

Ivanova, Ivona. «El Concepto de la tortura en relación con la traducción y la interpretación en los servicios públicos». FITISPos International Journal 2 (2015): 49-64.

Ivorra Pérez, Francisco Miguel. «How can Spanish Toy Manufacturers Achieve Intercultural Competence in English? A Contrastive Study of Spanish and U.S. Business Websites». CLINA 1.2 (2015): 87-97.

Jaime Pérez, Adriana. «Remote Interpreting in Public Services: Developing a 3G Phone Interpreting Application». Investigación emergente en Traducción e Interpretación. Eds. Raquel Lázaro Gutiérrez, María del Mar Sánchez Ramos y Francisco Vigier Moreno. Granada: Comares, 2015.

JANÉ LLIGÉ, JORDI. «Traducción, censura y construcción del discurso literario. La labor de los editores J. Janés, C. Barral y J. M. Castellet durante el franquismo». Traducción y censura: nuevas perspectivas. Eds. Gora Zaragoza Ninet, Juan José Martínez Sierra y José Javier Ávila-Cabrera. Quaderns de Filologia. Estudis Literaris 20 (2015): 73-90.

Jiménez GutiérRez, Isabel. «Discapacidad visual y estudios de Traducción e Interpretación. Dificultades de alumnos con discapacidad visual en las asignaturas de recursos informáticos aplicados a la Traducción e Interpretación y de traducción especializada». Traducimos desde el sur. VI Congreso Internacional de la AIETI. Ed. José Jorge Amigo Extremera. Las Palmas de Gran Canaria: Universidad de Las Palmas de Gran Canaria, 2015. 457-475.

Jordà MATHiasen, Eivor. «El cine como herramienta para la formación de traductores jurídicos». ESTUDIOS DE TRADUCCIÓN 5 (2OI5): I33-I47.

Kalina, Sylvia. «Ethical Challenges in Different Interpreting Settings». Insights in Interpreting. Status and Developments. Eds. Catalina Iliescu Gheorghiu y Juan Miguel Ortega Herráez. MonTI Special Issue 2 (2015): 63-86.

Killman, Jeff A. «Introducing Machine Translation in Translator Training: Comparing 'Information Mining' with Post-editing». ENTRECULTURAS 7-8 (2015): 179-193.

Kocbek, Alenka. «The Decalogue of Legal Translation - Contracts in Intercultural Legal Communication». CLINA 1.1 (2015): 71-86.

KuZnik, AnNa. «Prácticas, in-service training y transferencia del conocimiento. Formación de traductores e investigación en traducción, en contacto con la realidad del mercado». Traducimos desde el sur. VI Congreso Internacional de la AIETI. Ed. José Jorge Amigo Extremera. Las Palmas de Gran Canaria: Universidad de Las Palmas de Gran Canaria, 2015. 566-586.

Lasa Álvarez, Begoña. «Novela inglesa y censura inquisitorial durante el reinado de Fernando VII: un expediente de 1815-1816». Traducción y censura: nuevas perspectivas. Eds. Gora Zaragoza Ninet, Juan José Martínez Sierra y José Javier Ávila-Cabrera. Quaderns de Filologia. Estudis Literaris 20 (2015): 145-161.

Lázaro Gutiérrez, Raquel y Sánchez Ramos, María del Mar. «El uso de corpus en la formación de intérpretes en los servicios públicos en casos de violencia de género en España». Metodologías y aplicaciones en la investigación en traducción e interpretación con corpus. The Hermeneus Conference. Eds. María Teresa Sánchez Nieto, Susana Álvarez Álvarez; Verónica ArnáizUzquiza, Teresa Ortego Antón, Leticia Santamaría Ciordia y Rosa Fernández Muñiz. Valladolid: Universidad de Valladolid, 2015. 183-195.

Lázaro Gutiérrez y Vigier Moreno, Francisco, eds. Investigación Emergente en Traducción e Interpretación. Granada: Comares, 2015.

LeJeune, Pierre. «Traducir el sintagma nominal sujeto escueto del inglés: casos del español y del francés». Traducimos desde el sur. VI Congreso Internacional de la AIETI. Ed. José Jorge Amigo Extremera. Las Palmas de Gran Canaria: Universidad de Las Palmas de Gran Canaria, 2015. 404-420. 
LENGLET, CÉDRIC. «Norms in Face-threatening Instances of Simultaneous Conference Interpreting: Results from a Questionnaire». Insights in Interpreting. Status and Developments. Eds. Catalina Iliescu Gheorghiu y Juan Miguel Ortega Herráez. MonTI Special Issue 2 (2015): 237-258.

López Guix, JuAn Gabriel. «De espejos y máscaras. Una propuesta para la traducción de los lenguajes 'rotos'». TRANS (Revista de Traductología) 19.2 (2015): 265-276.

— «La traducción cuántica: Alicia en el país de las maravillas». Vasos comunicantes 46 (2015): 9-18.

Lucero García, Marta. «Hacia un habitus interseccional del traductor/intérprete en contextos de violencia de género». Construir puentes de comunicación en el ámbito de la violencia de género. I Congreso Internacional SOS-VICS. Eds. Maribel del Pozo Triviño, Carmen Toledano Buendía, David Casado-Neira y Doris Fernandes del Pozo. Granada: Comares, 2015. 83-92.

LuNG, RACHEL. «Solitude and Breakthroughs in Translation Studies Research». Hermeneus 17 (2015): 21-27.

Marcelo Wirnitzer, Gisela. «Hacia nuevos modelos literarios: traducción y tabúes en literatura infantil y juvenil». Traducimos desde el sur. VI Congreso Internacional de la AIETI. Ed. José Jorge Amigo Extremera. Las Palmas de Gran Canaria: Universidad de Las Palmas de Gran Canaria, 2015. 287-298.

Marín García, Álvaroy Romo Recio, Patricia. «Allá al fondo está el cliente, pero no tenga miedo: una visión empresarial de la evaluación de la calidad». Traducimos desde el sur. VI Congreso Internacional de la AIETI. Ed. José Jorge Amigo Extremera. Las Palmas de Gran Canaria: Universidad de Las Palmas de Gran Canaria, 2015. 553-565.

Martín Párraga, Javier. «Traduciendo a Joseph Addison: problemas y desafíos». ENTRECULTURAS 7-8 (2015): 35-47.

Martin, ANNe, «La formación en interpretación en España: pasado y presente». Insights in Interpreting. Status and Developments. Eds. Catalina Iliescu Gheorghiu y Juan Miguel Ortega Herráez. MonTI Special Issue 2 (2015): 87-110.

Martínez Martos, Isabel María y Corpas Pastor, Gloria. «En torno a la evaluación de la calidad en traducción turística. Un estudio de caso». Traducimos desde el sur. VI Congreso Internacional de la AIETI. Ed. José Jorge Amigo Extremera. Las Palmas de Gran Canaria: Universidad de Las Palmas de Gran Canaria, 2015. 701-718.

Martínez Mateo, Roberto. «Una revisión de la censura en la Literatura Infantil y Juvenil (LIJ) traducida del inglés en España desde la etapa franquista a la actualidad». Traducción y censura: nuevas perspectivas. Eds. Gora Zaragoza Ninet, Juan José Martínez Sierra y José Javier ÁvilaCabrera. Quaderns de Filologia. Estudis Literaris 20 (2015): 163-182.

Martínez Pleguezuelos, Antonio J. y González-Iglesias González, Juan David. «La identidad censurada: representación y manipulación de la homosexualidad en la obra Té y simpatía». Traducción y censura: nuevas perspectivas. Eds. Gora Zaragoza Ninet, Juan José Martínez Sierra y José Javier Ávila-Cabrera. Quaderns de Filologia. Estudis Literaris 20 (2015): 53-67.

Martínez Sierra, Juan José. «La traducción de Nigger en Django Desencadenado: Un enfoque ideológico». Traducción, ideología y poder en la ficción audiovisual. Eds. Frederic Chaume y Mabel Richart Marset. Prosopopeya. Revista de crítica contemporánea 9 (2014-2015): 81-98.

Mata Buil, Ana. «Análisis comparativo de la recepción poética de T. S. Eliot, Marianne Moore y Edna St. Vincent Millay». Hermeneus 17 (2015): 137-177.

Mayor, Carlos. «Mientras, en las Rocosas». Vasos comunicantes 46 (2015): 23-32.

Mazagatos Angulo, Cristina. «Análisis comparativo-contrastivo de la comisión de modernización del lenguaje jurídico y del Plain English Movement y su aplicación a la traducción de sentencias judiciales». Investigación emergente en Traducción e Interpretación. Eds. Raquel Lázaro Gutiérrez, María del Mar Sánchez Ramos y Francisco Javier Vigier Moreno. Granada: Editorial Comares, 2015. 
Medina Reguera, Ana y Ramírez Delgado, Cristina. «Metodología para evaluar la localización web en España. Primeros resultados aplicados a un corpus de sitios web de pymes agroalimentarias andaluzas». Metodologías y aplicaciones en la investigación en traducción e interpretación con corpus. The Hermeneus Conference. Eds. María Teresa Sánchez Nieto, Susana Álvarez Álvarez; Verónica Arnáiz-Uzquiza, Teresa Ortego Antón, Leticia Santamaría Ciordia y Rosa Fernández Muñiz. Valladolid: Universidad de Valladolid, 2015. 293-319.

MEIRIÑo Gómez, Jesús. «Men in Black y 'los hombres de negro': el lenguaje metafórico en el discurso económico». Traducimos desde el sur. VI Congreso Internacional de la AIETI. Ed. José Jorge Amigo Extremera. Las Palmas de Gran Canaria: Universidad de Las Palmas de Gran Canaria, 2015. 639-654.

MÉNDEZ GonZÁLEZ, RAMón. «Localización y cultura: comprender los videojuegos como referentes culturales». ENTRECULTURAS 7-8 (2015): 741-759.

- «Paratraducción de la pareja texto/imagen: mutilación y manipulación de paratextos lingüísticos e icónicos». Sendebar 26 (2015): 83-98.

Mendoza García, InMa. «La aceptabilidad de la traducción cultural en la literatura para la infancia: una propuesta conceptual y metodológica». Tonos Digital. Revista Electrónica de Estudios Filológicos (2015).

Merino Álvarez, Raquel. «Musicales traducidos y censurados en los escenarios españoles (19551985)». Traducción y censura: nuevas perspectivas. Eds. Gora Zaragoza Ninet, Juan José Martínez Sierra y José Javier Ávila-Cabrera. Quaderns de Filologia. Estudis Literaris 20 (2015): 219-235.

Meseguer Cutillas, Purificación. «La traducción como arma propagandística: censura de Orwell, Abellio y Koestler en la España franquista». Traducción y censura: nuevas perspectivas. Eds. Gora Zaragoza Ninet, Juan José Martínez Sierra y José Javier Ávila-Cabrera. Quaderns de Filologia. Estudis Literaris 20 (2015): 107-122.

MoJica LóPEZ, EsPeranZa. «La interpretación en el ámbito judicial en España en casos específicos de violencia de género». Investigación emergente en Traducción e Interpretación. Eds. Raquel Lázaro Gutiérrez, María del Mar Sánchez Ramos y Francisco Javier Vigier Moreno. Granada: Editorial Comares, 2015.

Morató, Yolanda. «Wyndham Lewis y su recepción en España: etapas editoriales y traducciones». Traducción y censura: nuevas perspectivas. Eds. Gora Zaragoza Ninet, Juan José Martínez Sierra y José Javier Ávila-Cabrera. Quaderns de Filologia. Estudis Literaris 20 (2015): 183-197.

Moreno Bello, Yolanda. «Interpreting at War: Fighting Language Manipulation». Investigación emergente en Traducción e Interpretación. Eds. Raquel Lázaro Gutiérrez, María del Mar Sánchez Ramos y Francisco Javier Vigier Moreno. Granada: Editorial Comares, 2015.

Muegge, Uwe. «Do Translation Standards Encourage Effective Terminology Management?» Normalització en la indústria de la traducció. Eds. Celia Rico Pérez y Willem Stoeller. Revista Tradumàtica 13 (2015): 552-560.

Muñoz Martín, Ricardo. «Traducimos desde el Sur. Celebración de un balance siempre provisional». Traducimos desde el sur. VI Congreso Internacional de la AIETI. Ed. José Jorge Amigo Extremera. Las Palmas de Gran Canaria: Universidad de Las Palmas de Gran Canaria, 2015. 11-17.

Muñoz Ramos, María y Corpas Pastor, Gloria. «El EEES y la competencia tecnológica: los nuevos grados en Traducción». Traducimos desde el sur. VI Congreso Internacional de la AIETI. Ed. José Jorge Amigo Extremera. Las Palmas de Gran Canaria: Universidad de Las Palmas de Gran Canaria, 2015. 509-523.

Naranjo Gutiérrez, Laura y Torres García, María Carmen. «A cuatro manos, a dos cabezas o cómo maximizar tu potencial (y el de la traducción)». Vasos comunicantes 46 (2015): 101-106.

Naredo Molero, María. «La interpretación especializada en violencia de género, una obligación de derechos humanos». Construir puentes de comunicación en el ámbito de la violencia de género. I Congreso Internacional SOS-VICS. Eds. Maribel del Pozo Triviño, Carmen Toledano Buendía, David Casado-Neira y Doris Fernandes del Pozo. Granada: Comares, 2015. 37-46. 
NiETo Flores, Marta. «De camino hacia una traducción post-positivista». ENTRECULTURAS 7-8 (2015): 95-114.

NovodvorsKi, Ariel. «Estudios de la traducción basados en corpus. Aspectos metodológicos para el análisis de estilo». Metodologías y aplicaciones en la investigación en traducción e interpretación con corpus The Hermeneus Conference. Eds. María Teresa Sánchez Nieto, Susana Álvarez Álvarez; Verónica Arnáiz-Uzquiza, Teresa Ortego Antón, Leticia Santamaría Ciordia y Rosa Fernández Muñiz. Valladolid: Universidad de Valladolid, 2015. 117-137.

Olalla Soler, Christian y Vert Bolaños, Olga. «Traducción y tecnología: herramientas del proceso traductor como actividad profesional. El punto de vista de los estudiantes». Normalització en la indústria de la traducció. Eds. Celia Rico Pérez y Willem Stoeller. Revista Tradumàtica 13 (2015): 623-640.

Olalla Soler, Christian, Sánchez Hita, Judith y Prado Junquera, Borja Manuel. «La percepción de los estudiantes de Traducción e Interpretación sobre los estudios en Traducción e Interpretación de España. Un estudio empírico». Sendebar 26 (2015): 99-135.

Oliver GonZÁlez, ANTONI. «TMX: Intercambio de memorias de traducción». Normalització en la indústria de la traducció. Eds. Celia Rico Pérez y Willem Stoeller. Revista Tradumàtica 13 (2015): 608-622.

Olvera lobo, María Dolores y Gutiérrez Artacho, Juncal. «Nuevas tendencias en Recuperación de Información: la Búsqueda de Respuestas desde la perspectiva de la traducción». Traducimos desde el sur. VI Congreso Internacional de la AIETI. Ed. José Jorge Amigo Extremera. Las Palmas de Gran Canaria: Universidad de Las Palmas de Gran Canaria, 2015. 604-620.

Olvera lobo, María Dolores, Gutiérrez Artacho, Juncal y Robinson-Fryer, Bryan-John. «Acciones formativas Web 2.0: el Grado en Traducción e Interpretación como banco de pruebas». Traducimos desde el sur. VI Congreso Internacional de la AIETI. Ed. José Jorge Amigo Extremera. Las Palmas de Gran Canaria: Universidad de Las Palmas de Gran Canaria, 2015. 524-539.

ORDóÑEZ LóPEZ, PILAR. «Investigación histórica y tendencias historiográficas contemporáneas. Hacia un reader de historiografía de la traducción». Traducimos desde el sur. VI Congreso Internacional de la AIETI. Ed. José Jorge Amigo Extremera. Las Palmas de Gran Canaria: Universidad de Las Palmas de Gran Canaria, 2015. 177-192.

Ordóñez López, Pilar y Sabio Pinilla, José Antonio, eds. Historiografía de la traducción en el espacio ibérico. Textos contemporáneos. Cuenca: Universidad de Castilla La Mancha, 2015.

Ortega Arjonilla, Emilio y San Ginés Aguilar, Pedro. «Bibliografía especializada sobre traducción e interpretación: La colección Interlingua de la editorial Comares de Granada (19962015)». ENTRECULTURAS 7-8 (2015): 871-902.

Ortega Herráez, Juan Miguel. «Reflexiones en torno al binomio formación-acreditación como elementos constitutivos de la profesionalización de la interpretación jurídica». La interpretación en entornos judiciales. Coord. Jesús Baigorri Jalón y Mariachiara Russo. TRANS (Revista de Traductología) 19.1 (2015): 131-152.

Ortiz SoRIANO, AdELA. «La imparcialidad en la interpretación policial». Legal Interpreting at a Turning Point. Eds. María Jesús Blasco Mayor y Maribel del Pozo Triviño. MonTI 7 (2015): 207-241.

Parra Galiano, Silvia. «El conocimiento experto (pericia) en la revisión de traducciones: clave en la gestión y propuestas de investigación». Traducimos desde el sur. VI Congreso Internacional de la AIETI. Ed. José Jorge Amigo Extremera. Las Palmas de Gran Canaria: Universidad de Las Palmas de Gran Canaria, 2015. 587-603.

Pascual Olaguíbel, Marina. «La interpretación en el Tribunal de Justicia de la Unión Europea». La interpretación en entornos judiciales. Coord. Jesús Baigorri Jalón y Mariachiara Russo. TRANS (Revista de Traductología) 19.1 (2015): 33-41.

PAVESI, María. «De Lei a Tu y viceversa: estudio de caso de la creatividad en traducción audiovisual». Eds. Frederic Chaume y Mabel Richart Marset. Prosopopeya. Revista de crítica contemporánea 9 (2014-2015): 111-134. 
Pegenaute Rodriguez, Luis. «Exiliados liberales decimononicos en Inglaterra: su labor como traductores literarios». Traducimos desde el sur. VI Congreso Internacional de la AIETI. Ed. José Jorge Amigo Extremera. Las Palmas de Gran Canaria: Universidad de Las Palmas de Gran Canaria, 2015. 206-216.

Penas IbáÑez, Beatriz. «The Role of Indirect Translation in the Ralentization of Cultural Modernization: The Intermediate Role of Hemingway's Early Spanish translations». Transfer. Revista electrónica sobre traducción e interculturalidad 10.1-2 (2015): 51-74.

PÉrez Freire, Silvia. «Los/las intérpretes vistos por los/las expertas y por las víctimas». Construir puentes de comunicación en el ámbito de la violencia de género. I Congreso Internacional SOSVICS. Eds. Maribel del Pozo Triviño, Carmen Toledano Buendía, David Casado-Neira y Doris Fernandes del Pozo. Granada: Comares, 2015. 101-112.

Pérez-Luzardo Díaz, Jessica. «The Style Parameter in Quality Evaluation and Expectations of Simultaneous Interpreting». Sendebar 26 (2015): 213-233.

Petersen, Lucas. «Ulises inmigrante: Salas Subirat, sus orígenes catalanes, su argentinidad incómoda». 1611 (Revista de Historia de la Traducción) 9 (2015).

Pöchacker, Franz. «Los estudios de interpretación, enfocados desde el Sur». Traducimos desde el sur. VI Congreso Internacional de la AIETI. Ed. José Jorge Amigo Extremera. Las Palmas de Gran Canaria: Universidad de Las Palmas de Gran Canaria, 2015. 27-38.

Pons Alorda, Jaume C. «Més enllà de la traducció de Fulles d'herba de Walt Whitman». 1611 (Revista de Historia de la Traducción) 9 (2015).

Pozo Triviño, Maribel del y Toledano Buendía, Carmen. «El proyecto Speak Out for Support (SOS-VICS), un paso adelante en la comunicación con víctimas de violencia de género a través de intérpretes». Construir puentes de comunicación en el ámbito de la violencia de género. I Congreso Internacional SOS-VICS. Eds. Maribel del Pozo Triviño, Carmen Toledano Buendía, David Casado-Neira y Doris Fernandes del Pozo. Granada: Comares, 2015. 1-17.

Pozo Triviño, Maribel del, Toledano Buendía, Carmen Casado-Neira, David y Fernandes DEL Pozo, Doris, eds. Construir puentes de comunicación en el ámbito de la violencia de género. Granada: Comares. 2015, 1-152.

Remael, Aline, van waes, luuk y Leijten, Mariëlle. «La subtitulación en directo con reconocimiento de voz: cómo determinar los retos». Traducción, ideología y poder en la ficción audiovisual. Eds. Frederic Chaume y Mabel Richart Marset. Prosopopeya. Revista de crítica contemporánea 9 (2014-2015): 233-264.

Rica Peromingo, Juan Pedro y Braga Riera, Jorge. Herramientas y técnicas para la traducción inglés-español: Los textos literarios. Madrid: Escolar y Mayo, 2015.

Richart MARSET, Mabel. «La censura de la corrección política: la traducción audiovisual a escena». Traducción y censura: nuevas perspectivas. Eds. Gora Zaragoza Ninet, Juan José Martínez Sierra y José Javier Ávila-Cabrera. Quaderns de Filologia. Estudis Literaris 20 (2015): 237-257.

Rico Pérez, Celia y Stoeller, Willem, eds. Normalització en la indústria de la traducció. Revista Tradumàtica 13 (2015): 539-640.

Roales Ruiz, ANTONio. «Los programas informáticos de subtitulación en la enseñanza / aprendizaje de la TAV dentro del ámbito universitario (1): una propuesta de evaluación». ENTRECULTURAS 7-8 (2015): 761-774.

- «Los programas informáticos de subtitulación en la enseñanza / aprendizaje de la TAV dentro del ámbito universitario (2): Análisis del software profesional». ENTRECULTURAS 7-8 (2015): 775-789.

Rodríguez Arcos, Irene. «La traducción ante los nuevos retos de la sociedad global». ENTRECULTURAS 7-8 (2015): 115-136.

Rodríguez CASTRO, MónICA. «Intrinsic and Extrinsic Sources of Translator Satisfaction: An Empirical Study». ENTRECULTURAS 7-8 (2015): 195-229. 
Rodríguez Herrera, José Manuel. «Shakespeare's Legal Wit: Evolution of the Translation of Shakespeare's Legal Puns into Spanish from the 20th to the 21st Century». Revista alicantina de Estudios Ingleses 28 (2015): 165-181.

Rodríguez Martínez, Manuel CRISTóbal. «Competencias y recursos para la práctica eficiente de la traducción profesional (Parte I)». ENTRECULTURAS 7-8 (2015): 231-257.

- «Competencias y recursos para la práctica eficiente de la traducción profesional (Parte II)». ENTRECULTURAS 7-8 (2015): 259-277.

RodrígueZ-TAPIA, SERgio. «Estrategias de traducción inglés-español basadas en el análisis cuantitativo de procedimientos de reformulación formal y conceptual del texto semiespecializado». Tonos Digital. Revista Electrónica de Estudios Filológicos 29 (2015).

Roiss, Silvia. «El Trabajo de Fin de Grado en el Grado de Traducción e Interpretación: evaluación de competencias y contenidos asociados al título. Estudio analítico y consecuencias didácticas». Quaderns. Revista de Traducció 22 (2015): 273-288.

Rojo López, Ana María y Meseguer Cutillas, Purificación. «Fomentando la creatividad: una propuesta didáctica para el aula de traducción». Quaderns. Revista de Traducció 22 (2015): 255-271.

Romero Fresco, Pablo. «Cine accesible: uniendo los puntos entre traducción audiovisual y la realización cinematográfica». Traducción, ideología y poder en la ficción audiovisual. Eds. Frederic Chaume y Mabel Richart Marset. Prosopopeya. Revista de crítica contemporánea 9 (2014-2015): 163-192.

Ruano San Segundo, Pablo. «El Pickwick de Galdós. Estudio traductológico de los verbos de habla a través de una aproximación computacional». Hermeneus 17 (2015): 209-231.

Ruiz Mezcua, Aurora. «¿Cómo elegir las herramientas de interpretación simultánea más apropiadas para la enseñanza?». Traducimos desde el sur. VI Congreso Internacional de la AIETI. Ed. José Jorge Amigo Extremera. Las Palmas de Gran Canaria: Universidad de Las Palmas de Gran Canaria, 2015. 105-118.

— «Aplicación práctica de la interpretación judicial a las aulas universitarias». Quaderns. Revista de Traducció 22 (2015): 307-323.

Ruiz Rosendo, Lucía. «Retos en la adquisición de destrezas en interpretación simultánea: estudio experimental sobre la evolución del alumno principiante». Traducimos desde el sur. VI Congreso Internacional de la AIETI. Ed. José Jorge Amigo Extremera. Las Palmas de Gran Canaria: Universidad de Las Palmas de Gran Canaria, 2015. 75-93.

Sánchez Nieto, María Teresa, Álvarez Álvarez, Susana, Arnaiz Urquiza, Verónica, Ortego, María Teresa, Santamaría Ciordia, Leticia, y Fernández Muñiz, Rosa. «Testimonios de la evolución de los estudios traductológicos basados en corpus durante la última década (20032013)». Metodologías y aplicaciones en la investigación en traducción e interpretación con corpus. The Hermeneus Conference. Eds. María Teresa Sánchez Nieto, Susana Álvarez Álvarez; Verónica Arnáiz-Uzquiza, Teresa Ortego Antón, Leticia Santamaría Ciordia y Rosa Fernández Muñiz. Valladolid: Universidad de Valladolid, 2015. 17-30.

- eds. Metodologías y aplicaciones en la investigación en traducción e interpretación con corpus. Valladolid: Universidad de Valladolid, 2015. 1-371.

SANDRElli, AnNalisa. «Becoming an Interpreter: The Role of Computer Technology». Insights in Interpreting. Status and Developments. Eds. Catalina Iliescu Gheorghiu y Juan Miguel Ortega Herráez. MonTI Special Issue 2 (2015): 111-138.

Santana Falcón, María del Mar y García Álvarez, Ana María. «Errores de traducción de realia en menús de Gran Canaria». Traducimos desde el sur. VI Congreso Internacional de la AIETI. Ed. José Jorge Amigo Extremera. Las Palmas de Gran Canaria: Universidad de Las Palmas de Gran Canaria, 2015. 683-700.

Santana, Mario. «Translation and literatures in Spain, 2003-2012». 1611 (Revista de Historia de la Traducción) 9 (2015). 
Socorro Trujillo, Karina. «Clasificación de textos comerciales en función de su grado de complejidad: una ayuda para la enseñanza de traducción». Traducimos desde el sur. VI Congreso Internacional de la AIETI. Ed. José Jorge Amigo Extremera. Las Palmas de Gran Canaria: Universidad de Las Palmas de Gran Canaria, 2015. 540-552.

Talaván, Noa, Rodríguez-Arancón, Pilar y Martín-Monje, Elena. «The Enhancement of Speaking Skills Practice and Assessment in an Online Environment». Tendencias en Educación y Lingüística. Coords. Lucía Pilar Cancelas y Ouviña, y Susana Sánchez Rodriguez. Cádiz: Editorial GEU, 2015. 329-351.

TAmaYo, ANA. «Estudio descriptivo de la subtitulación para niños sordos y con discapacidad auditiva en las cadenas infantiles y juveniles en España». Quaderns. Revista de Traducció 22 (2015): 363-383.

Toledano Buendía, Carmen, Abril Martí, María Isabel, del Pozo Triviño, María Isabel y Aguilera Ávila, Laura. «Hacia una especialización en interpretación en el ámbito de la violencia de género: investigación, formación y profesionalización». Insights in Interpreting. Status and Developments. Eds. Catalina Iliescu Gheorghiu y Juan Miguel Ortega Herráez. MonTI Special Issue 2 (2015): 139-160.

TORREJón, ENRIQUE. «La integración de tecnologías de la traducción: ¿bendición o maldición?». Normalització en la indústria de la traducció. Eds. Celia Rico Pérez y Willem Stoeller. Revista Tradumàtica 13 (2015): 561-570.

Torres del Rey, Jesús y Morado Vázquez, Lucía. «XLIFF, XML Localisation Interchange File Format, Translators, Localisation Standards». Normalització en la indústria de la traducció. Eds. Celia Rico Pérez y Willem Stoeller. Revista Tradumàtica 13 (2015): 584-607.

Tsaousi, AikAterinI. «Making Sound Accessible. The Labelling of Sound Effects in Subtitling for the Deaf and Hard-of-hearing». Hermeneus 17 (2015): 233-252.

VaAmonde Liste, Antonio. «Evaluación de las necesidades formativas de los/las intérpretes en el marco de la asistencia a las mujeres extranjeras víctimas de violencia de género (encuesta Delphi)». Construir puentes de comunicación en el ámbito de la violencia de género. I Congreso Internacional SOS-VICS. Eds. Maribel del Pozo Triviño, Carmen Toledano Buendía, David Casado-Neira y Doris Fernandes del Pozo. Granada: Comares, 2015. 125-134.

Valero Cuadra, Pino. «Las traducciones al español del bestseller alemán David Safier... y el caso de un soneto de Shakespeare traducido ad hoc». Traducimos desde el sur. VI Congreso Internacional de la AIETI. Ed. José Jorge Amigo Extremera. Las Palmas de Gran Canaria: Universidad de Las Palmas de Gran Canaria, 2015. 251-262.

Valero Garcés, Carmen y Kadric, Mira. «Multilingualism and TIPSI. A Shared European Challenge». Traducimos desde el sur. VI Congreso Internacional de la AIETI. Ed. José Jorge Amigo Extremera. Las Palmas de Gran Canaria: Universidad de Las Palmas de Gran Canaria, 2015. 39-51.

Valero Garcés, Carmen, Kadric, Mira, Schnell, Bettina, Rodríguez, Nadia y Cuñado, FERNANDO. «Estudio preliminar sobre el ejercicio de la interpretación y traducción judicial en España». Sendebar 26 (2015): 137-166.

VALERO GISBERT, MARÍA. «Reflexiones sobre la creación de la imagen mental a través de la audiodescripción (AD)». Traducción, ideología y poder en la ficción audiovisual. Eds. Frederic Chaume y Mabel Richart Marset. Prosopopeya. Revista de crítica contemporánea 9 (2014-2015): 219-232.

Vegara Fabregat, Laura. «Traducción y metáfora: Estudio de metáforas cognitivas del Tribunal Supremo de los Estados Unidos». Quaderns. Revista de Traducció 22 (2015): 325-346.

Vidal Claramonte, María Carmen África. «Hacia una traducción 'escribible': Between, de Christine Brooke-Rose». CLINA 1.1 (2015): 87-98.

VILAR SÁNChez, KARIN. «Análisis cualitativo de textos con un programa CAQDAS». Metodologías y aplicaciones en la investigación en traducción e interpretación con corpus. The Hermeneus Conference. Eds. María Teresa Sánchez Nieto, Susana Álvarez Álvarez; Verónica Arnáiz- 
Uzquiza, Teresa Ortego Antón, Leticia Santamaría Ciordia y Rosa Fernández Muñiz. Valladolid: Universidad de Valladolid, 2015. 271-291.

Villa Jiménez, Rosalía. «Análisis pragmático-cognitivo de tres versiones en español de The Tell-Tale Heart de Edgar Allan Poe». Hikma 14 (2015): 141-165.

Villalba JimÉneZ, RubÉN. «La medicina en televisión: implicaciones para la traducción. El caso del doblaje de las series sobre médicos». Sendebar 26 (2015): 9-36.

Wallace, Melissa. «Access and Protection: Civil Court Remedies for Victims of Domestic Violence and the Current State of Free Language Mediation in US Courts». Construir puentes de comunicación en el ámbito de la violencia de género. I Congreso Internacional SOS-VICS. Eds. Maribel del Pozo Triviño, Carmen Toledano Buendía, David Casado-Neira y Doris Fernandes del Pozo. Granada: Comares, 2015. 73-82.

— «Resisting Market Disorder and Ensuring Public Trust: Reimagining National Registers for Legal Interpreters in the United States and the European Union». Legal Interpreting at a Turning Point. Eds. María Jesús Blasco Mayor y Maribel del Pozo Triviño. MonTI 7 (2015): 115-140.

- «Current Dilemmas in Court Interpreting: Improving Quality and Access Through Smarter Testing and Administration Protocols». Insights in Interpreting. Status and Developments. Eds. Catalina Iliescu Gheorghiu y Juan Miguel Ortega Herráez. MonTI Special Issue 2 (2015): 217-236.

Wehrmeyer, Ella. «An Annotation System for Signed Language Interpreting Corpora». Hermeneus 17 (2015): 279-317.

Wu, Pei Chuan. «Asegurar la pertinencia de los objetos de estudio en traducción literaria: Una reflexión metodológica». Traducimos desde el sur. VI Congreso Internacional de la AIETI. Ed. José Jorge Amigo Extremera. Las Palmas de Gran Canaria: Universidad de Las Palmas de Gran Canaria, 2015. 331-348.

Zaragoza Ninet, Gora, Martínez Sierra, Juan José y Ávila-Cabrera, José Javier, eds. Traducción y censura: nuevas perspectivas. Quaderns de Filologia. Estudis Literaris 20 (2015): $1-257$.

— «Introducción». Traducción y censura: nuevas perspectivas. Eds. Gora Zaragoza Ninet, Juan José Martínez Sierra y José Javier Ávila-Cabrera. Quaderns de Filologia. Estudis Literaris 20 (2015): 9-13.

Zarandona Fernández, Juan Miguel. «The Nun's Story / Historia de una monja: el Congo de Kathryn Hulme, Fred Zinnemann y Audrey Hepburn y sus traductores al español». Traducimos desde el sur. VI Congreso Internacional de la AIETI. Ed. José Jorge Amigo Extremera. Las Palmas de Gran Canaria: Universidad de Las Palmas de Gran Canaria, 2015. 263-280.

Zaro Vera, JuAn Jesús. «Traducción y propaganda religiosa: Gibraltar y la labor traductora de William Harris Rule». Quaderns. Revista de Traducció 22 (2015): 135-148. 


\title{
F. CULTURA Y CINE
}

M. ${ }^{a}$ Luz Arroyo VÁzQuez

UNED

larroyo@flog.uned.es Antonia Sagredo Santos

UNED

asagredo@ flog.uned.es

\section{Índice general}

\author{
1. CULTURA
}

2. CINE

\section{CUltura}

Aguirre, Nancy A. «Exile and Repatriation in the 'Barrios': the Great Depression in La Prensa and La Opinión, 1930-1932». Camino Real. Estudios de las hispanidades norteamericanas 10 (2015): 93-108.

Alsina Rísquez, Cristina. «Dissent as Therapy: The Case of the Veterans of the American War in Vietnam». Atlantis. Journal of the Spanish Association of Anglo-American Studies 37.2 (2015): 101-119.

Ameal Pérez, Alberto Jorge. «Nicanor Bolet Pereza en La revista ilustrada de Nueva York (18851890)». Camino Real. Estudios de las Hispanidades Norteamericanas 10 (2015): 21-37.

Arroyo Vázquez, M. ${ }^{a}$ Luz. «The Empowerment of American Women During the Great Depression in Comparative Perspective». RIAS. Review of International American Studies 7.2 (2014): 141-156.

- «Mujeres en los altos cargos de la política estadounidense contemporánea: su presencia en los gabinetes presidenciales». Pensar con la historia desde el siglo XXI. Eds. Pilar Folguera, Juan Carlos Pereira et al. Madrid: Universidad Autónoma Ediciones, 2015. 5545-5563.

Bartley, Leanne, e Hidalgo-Tenorio, Encarnación. «Constructing Perceptions of Sexual Orientation: A Corpus-based Critical Discourse Analysis of Transitivity in the Irish Press». Estudios irlandeses. Journal of Irish Studies 10 (2015): 14-34.

Bosch, Aurora. «Cincuenta años después de la 'lucha contra la pobreza': socialismo y liberalismo en los sesenta estadounidenses». Pensar con la historia desde el siglo XXI. Eds. Pilar Folguera, Juan Carlos Pereira et al. Madrid: Universidad Autónoma Ediciones, 2015. 1995-2009.

— «Un consenso conflictivo: 'Liberales contra liberales' en Estados Unidos, 1946-1948». Historia social 79 (2014): 43-60.

- «La segunda declaración de derechos: ciudadanía social y redefinición democrática en Estados Unidos, 1932-1946». Caminos de democracia: ciudadanías y culturas democráticas en el siglo XX. Coords. Ana M. Aguado y Luz Sanfeliu. Granada: Comares, 2014. 63-79. 
Casavantes Bradford, Anita. "'La Conciencia del Gran Miami’: Monsignor Bryan Walsh, Cold War Catholicism, and the Politics of Asylum in Multiethnic Miami». Camino Real. Estudios de las Hispanidades Norteamericanas 10 (2015): 147-159.

Castro Arcos, Javier. «Estados Unidos y la guerra por el desarrollo: el control de la natalidad en Chile, 1960-1970». Revista Complutense de Historia de América 41 (2015): 95-120.

Chastagner, Claude. «'Charanga Cakewalk’: Tejano Music Takes Center Stage». Estudios ingleses de la Universidad Complutense 23 (2015): 83-96.

Claramonte Arrufat, Jordi. Desacoplados. Estética y política del western. Hamlet: el príncipe de los cercamientos. Madrid: Servicio de Publicaciones de la UNED, 2015.

Crowley, Tony. «Hegemonic Shifts: The Latest from the Walls of Northern Ireland». Estudios irlandeses. Journal of Irish Studies 10 (2015): 58-76.

- «Modernizadores y tecnócratas. Estados Unidos ante la política educativa y científica de la España del desarrollo». Historia y política: ideas, procesos y movimientos sociales 34 (2015): 113-146.

De Gregorio-Godeo, Eduardo, y Mateos-Aparicio Martín-Albo, Ángel, eds. Constructing Selves: Issues in Gender, Age, Ethnicity and Nation. Cuenca: Ediciones de la Universidad de Castilla-La Mancha, 2015.

Delgado Gómez-Escalonilla, Lorenzo. «La diplomacia pública de Estados Unidos. Una perspectiva histórica». Revista Complutense de Historia de América 40 (2014): 277-301.

Dessens, Natalie. «The Sounds of Babel: Staging American Ethnic Diversity in Early NineteenthCentury New Orleans». Estudios ingleses de la Universidad Complutense 23 (2015): 15-27.

Díaz Geada, Aba y Lanero Táboas, Daniel. «Modelos de modernización para el desarrollismo: el influjo de las propuestas estadounidenses en el Servicio de Extensión Agraria (1955-1975)». Revista Complutense de Historia de América 41 (2015): 71-94.

Fernández Rodríguez, Carolina. «Latina Super-Heroines: Hot Tamales in Tights vs. Women Warriors, Wrestlers and Guerrilla Fighters of La Raza». Complutense Journal of English Studies 25 (2015): 115-136.

Feu, MonserRat. «Sergio Aragonés Marginalizes Francoism in the Exile Newspaper España Libre (NYC).» Camino Real. Estudios de las Hispanidades Norteamericanas 10 (2015): 129-146.

Fotia, LaURA. «Proyección y política cultural estadounidense en Argentina, (1928-1941)». Revista Complutense de Historia de América 41 (2015): 21-46.

Fombuena Borrás, Rosario. «Las mujeres del partido republican en los Estados Unidos», en Pilar Folguera y Juan Carlos Pereira et al. eds. Pensar con la historia desde el siglo XXI. Madrid: Universidad Autónoma Ediciones, 2015. 5545-5563.

García Gómez, Antonio y Garrido Pozo, Fátima. «The Boundaries of Language and Gender Revisited: Performing Gender in Social Networks.» Odisea. Revista de Estudios Ingleses 16 (2015): 39-58.

Garrido Castellano, Carlos. «Después de la unidad en la diversidad. Identidad, nación y arte indio contemporáneo». Identidades en contexto y cultura posmoderna. Ensayos críticos. eds. Ángel Mateos-Aparicio Martín-Albo y Eduardo de Gregorio-Godeo. Oviedo: KRK Ediciones, 2015. 89-94.

Gómez Blesa, Mercedes. «Las intelectuales republicanas: la conquista de la ciudadanía». La residencia de señoritas y otras redes culturales femeninas. Coords. Josefina Cuesta Bustillo et al. Salamanca: Ediciones Universidad de Salamanca, 2015. 253-266.

Houliston, Victor. «Filling in the Blanks: Catholic Hopes for the English Succession». The SEDERI Yearbook 25 (2015): 77-104.

Huguet Santos, Montserrat. «Tradición misional y legado de las educadoras estadounidenses en España». La residencia de señoritas y otras redes culturales femeninas. Coords. Josefina Cuesta Bustillo et al. Salamanca: Ediciones Universidad de Salamanca, 2015. 71-116. 
Joyce, Valerie. «(Dis)Embodied Voices’ and (Dis)Appearing Dialects: Staging a Living Historiography of Early African American Women». Estudios ingleses de la Universidad Complutense 23 (2015): 29-42.

Karatzas, Konstantinos D. «Analyze the Strengths and Limitations of Nonviolence as Practiced by King and the Civil Rights Movement». English and American Studies in Spain: New Developments and Trends. Eds. Alberto Lázaro Lafuente y María Dolores Porto Requejo. Alcalá de Henares: Servicio de Publicaciones de la Universidad de Alcalá, 2015 (libro electrónico). 99-105.

Keating, Antony. «Administrative Expedience and the Avoidance of Scandal: Ireland's Industrial and Reformatory Schools and the Inter-Departmental Committee of 1962-3». Estudios irlandeses. Journal of Irish Studies 10 (2015): 95-108.

Kinnally, Cara Anne. «Race, Slavery, and Liberalism in Lorenzo de Zavala's Viage a los EstadosUnidos del Norte de América». Camino Real. Estudios de las Hispanidades Norteamericanas 10 (2015): 77-91.

López Ruiz, Baltasar. «European Immigration in Western Canada: Acculturation Processes within the Francophone Communities in the Canadian Prairies». Revista Internacional de Estudios Migratorios (RIEM) 5.1 (2015): 61-92.

NiÑo RodRíGUEZ, ANTONIO. «El modelo de modernización estadounidense y sus efectos en España y en América Latina». Revista Complutense de Historia de América 41 (2015): 15-19.

Partyka, Joanna. «Mad Madge dos veces locas: la científica revolucionaria del siglo XVII». Locas. Escritoras y personajes femeninos cuestionando las normas. Eds. Milagro Martín Clavijo et al. Sevilla: Arcibel Editores, 2015. 1173-1180.

Peñalba García, Mercedes. «Writing the Self, Drawing the Self: Identity and Self-Reflexivity in Craig Thompson Graphic Memoir Blankets». Cultura, lenguaje y representación 14 (2015): 155-180.

PÉrez, LisANDro. «The Schism of 1868 and the Growth of Cuban New York.» Camino Real. Estudios de las Hispanidades Norteamericanas 10 (2015): 61-76.

Poey, Delia. «To the Beat of Their Own Drum: Women in Salsa». Estudios ingleses de la Universidad Complutense 23 (2015): 69-81.

Sagredo Santos, Antonia. «La aproximación militar entre España y Estados Unidos en pleno escalonamiento de la Guerra Fría: su eco en la prensa estadounidense (1947-1953)». Pensar con la historia desde el siglo XXI. Eds. Pilar Folguera, Juan Carlos Pereira et al. Madrid: Universidad Autónoma Ediciones, 2015. 597-616.

- «La formación de la identidad irlandesa en los Estados Unidos». Identidades en contexto y cultura posmoderna. Ensayos críticos. Eds. Ángel Mateos-Aparicio Martín-Albo y Eduardo de GregorioGodeo. Oviedo: KRK Ediciones, 2015. 105-114.

- «Mujeres que desafiaron las normas y costumbres de la política estadounidense». Locas. Escritoras y personajes femeninos cuestionando las normas. Eds. Milagro Martín Clavijo et al. Sevilla: Arcibel Editores, 2015. 1495-1509.

SÁnchez Román, José Antonio. «El multilateralismo como intervencionismo. Estados Unidos y la Sociedad de Naciones en América Latina (1930-1946)». Revista Complutense de Historia de América 41 (2015): 47-69.

Santaemilia Ruiz, José. «Translating Sex(uality) from English into Spanish and Vice-versa: a Cultural and Ideological Challenge.» Atlantis. Revista de la Asociación Española de Estudios Anglo-Norteamericanos 37.1 (2015): 139-156.

Segura García, Teresa. «Maharajás y modernidad: un proyecto de reformismo hindú en un principado de la India, 1910-1940». Identidades en contexto y cultura posmoderna. Ensayos críticos. Eds. Ángel Mateos-Aparicio Martín-Albo y Eduardo de Gregorio-Godeo. Oviedo: KRK Ediciones, 2015. 77-87.

Sell, Roger D. «Pinter, Herbert, Dickens: Post-postmodern Communicational Studies and the Humanities». English and American Studies in Spain: New Developments and Trends. Eds. Alberto 
Lázaro Lafuente y María Dolores Porto Requejo. Alcalá de Henares: Servicio de Publicaciones de la Universidad de Alcalá, 2015 (libro electrónico). 31-43.

Stefani, AnNE. «Sounds of Freedom: Songs in the 1960s Southern Civil Rights Movement». Estudios ingleses de la Universidad Complutense 23 (2015): 55-67.

Sugars, Cynthia. "'Canadian, please': The Intimate Space of YouTube Racism». Canada and Beyond: A Journal of Canadian Literary and Cultural Studies 5 (2015): 50-71.

Torres Marquínez, Catalina. «Women's Network behind Frances Perkins's Appointment». Revista de Estudios Norteamericanos 19 (2015): 151-166.

Van Hoy, Teresa. «Mexican Exiles and the Monroe Doctrine, New York and the Borderlands, 1865». Camino Real. Estudios de las Hispanidades Norteamericanas 10 (2015): 39-60.

Varela Lago, Ana. «From migrants to Exiles: the Spanish Civil War and the Spanish Immigrant Communities in the United States.» Camino Real. Estudios de las Hispanidades Norteamericanas 10 (2015): 111-128.

\section{CINE Y TELEVISIÓN}

BARdÉs, CÉSAR. El sueño americano. El cine en la Era Kennedy. Madrid: Quadrivium, 2015.

Bartolomé Leal, ANDrÉs. «Strangers in a Strange Land: Cinema, Identity and the Modern NationState in Roman Polanski's The Ghost Writer». Atlantis. Journal of the Spanish Association of Anglo-American Studies 37.2 (2015): 121-138.

Bueno Alonso, Jorge Luis. "That's what I like about Shakespeare; it's the Pictures': A Teaching-Oriented Approach to Shakespearean Textual Analysis through Film (with Special Reference to Macbeth V.5.15-28)». Babel afial: Aspectos de filología inglesa y alemana 24 (2015): 131-144.

Carrasco-Carrasco, Rocío. «The Gendering of Technology in Science Fiction Cinema: the Sensitive Male Robot». English and American Studies in Spain: New Developments and Trends. Eds. Alberto Lázaro Lafuente y María Dolores Porto Requejo. Alcalá de Henares: Servicio de Publicaciones de la Universidad de Alcalá, 2015 (libro electrónico). 76-83.

Codó Martínez, Jordi. «Transnationalism and the Decentralization of the Global Film Industry». Coolabah 16 (2015): 34-47.

Cousins, Mark. Historia del Cine. Madrid: Blume, 2015.

CURIESES, ÓsCAR. «Lulu on the Bridge de Paul Auster, una reelaboración cinematográfica del mito de Pandora a través del sueño». $452^{\circ}$. Revista de teoría de la literatura y literatura comparada 12 (2015): 199-212.

Donapetry, María. «Sentido y sensibilidad de Sally Potter en Yes». Babel afial: Aspectos de filología inglesa y alemana 24 (2015): 109-130.

ECheverría Domingo, Julia. «Liquid Cinematography and the Representation of Viral Threats in Alfonso Cuarón's Children of Men». Atlantis. Journal of the Spanish Association of AngloAmerican Studies 37.2 (2015): 139-155.

Gómez-Moreno, Marta. «La influencia del arquetipo la gran madre en el bullying a través de Carrie (1974) de Stephen King». Feminismos 25 (2015): 159-182.

Hanrahan, Fiedhlim. «The Poverty Tour: Life in the Slums of Mumbai and Manila as Seen in Danny Boyle's Slumdog Millionaire and Merlinda Bobis's The Solemn Lantern Maker». Atlantis. Journal of the Spanish Association of Anglo-American Studies 37.1 (2015): 101-119.

JuÁrez Hervás, Luisa. «La americanización del Holocausto como fenómeno transnacional: Video Fortunoff Archive en Yale University, Steven Spielberg Film and Video Archive en el USHMM de Washington, USC Shoa Foundation Visual History Archive Online y la identidad de la segunda generación». English and American Studies in Spain: New Developments and Trends. Eds. Alberto 
Lázaro Lafuente y María Dolores Porto Requejo. Alcalá de Henares: Servicio de Publicaciones de la Universidad de Alcalá, 2015 (libro electrónico). 91-98.

Kennedy, TodD. «On the Road to 'Some' Place: Sofia Coppola's Dissident Modernism Against a Postmodern Landscape». Miscelánea 52 (2015): 51-67.

Koster, Serge. Las fascinantes rubias de Alfred Hitchock. Madrid: Periférica, 2015.

Lapeña Marchena, Óscar. «La contaminación de géneros en el cine sobre el Mundo Antiguo». Trocadero. Revista de Historia Moderna y Contemporánea 27 (2015): 151-172.

Lena, Alberto. Alfred Hitchkock, Thornton Wilder y 'La sombra de una duda'. Valladolid: Ediciones de la Universidad de Valladolid, 2015.

Orán Llarena, Fabián. «Ridley Scott's Dystopia Meets Ronald Reagan's America: Class Conflict and Political Disclosure in Blade Runner: The Final Cut». Revista canaria de estudios ingleses 70 (2015): 146-158.

Orellana, Juan. Cine e Ideología. Madrid: Stella Maris, 2015.

Parrondo Carretero, Concepción. «The Necessity of Agency in The Secret Life of Bees, the Film». English and American Studies in Spain: New Developments and Trends. Eds. Alberto Lázaro Lafuente y María Dolores Porto Requejo. Alcalá de Henares: Servicio de Publicaciones de la Universidad de Alcalá, 2015 (libro electrónico). 129-135.

Rodríguez Salas, Gerardo. «The Walking Dead: A Communitarian Study». Verbeia. Revista de estudios filológicos 0 (2015): 275-285.

Sánchez Palencia, Carolina. «'The tropics make it difficult to mope': The Imaginative Geography of Alexander Payne's The Descendants (2011)». IJES: International Journal of English Studies 15.2 (2015): 81-95.

Valero Redondo, María. «Giving Voice to the Subaltern: Ideology and Socio-Historical Factors in Andrea Arnold's Adaptation of Wuthering Heights». English and American Studies in Spain: New Developments and Trends. Eds. Alberto Lázaro Lafuente y María Dolores Porto Requejo. Alcalá de Henares: Servicio de Publicaciones de la Universidad de Alcalá, 2015 (libro electrónico). 144-150. 